\title{
邢窑的科学研究
}

\section{鲁晓珂 ${ }^{(1)} ，$ 李伟东 ${ }^{(1)}$, 罗宏杰 ${ }^{1}$ ，韩立森 ${ }^{2}$ ，王会民 ${ }^{(2)}$, 李强 ${ }^{(1)}$}

(1) 中国科学院上海硅酸盐研究所, 古陶瓷科学研究国家文物局重点科研基地, 上海 200050;

(2) 河北省文物研究所, 石家庄 050031

*E-mail: luxiaoke@mail.sic.ac.cn

收稿日期: 2012-02-15; 接受日期: 2012-05-18

国家文物局“指南针计划”(批准号: 20100301)、国家重点基础研究发展计划(“973”计划)(批准号: 2012CB720901)、国家自然科学基金(批 准号: 51072220)和国家质检公益性行业科研专项(批准号: 201110243)资助项目

\begin{abstract}
摘要邢窑是我国北方古代的著名窑场, 以生产白瓷著称于世, 其兴盛于隋唐, 至五代走向 衰落, 而考古界大多认为, 金代的邢窑基本属于定窑系. 本研究通过对河北邢窑遗址出土的 470 余件瓷器标本的化学组成、烧成温度、釉面白度以及物理性能的系统测试与分析, 首次从 科学技术角度较为全面的反映了邢窑自隋代至金代的发展演变过程, 这对于补充和完善中国 古陶瓷科技发展史具有非常重要的意义.

关键词

邢窑

白瓷

统计分析 制瓷工艺
\end{abstract}

邢窑以生产白瓷著称于世, 邢窑白瓷开创了我 国瓷器发展史上“南青北白”的局面. 自 20 世纪 80 年 代初开始, 其遗址在河北省临城、内丘、邢台等地区 相继被发现. 邢窑考古队曾于 1988 年至 1991 年间对 内丘城关和临城祁村两处规模较大的遗址进行了试 掘, 而在内丘城关西关北窑址的隋代灰坑中出土了 一批具有透影性能、胎体厚度小于 $1 \mathrm{~mm}$ 的薄胎精细 白瓷器, 被称之为“透影白瓷”, 其精湛的工艺技术水 平引起了学术界的极大关注. 内丘城关西关北窑址 位于李阳河东南岸的台地上, 考古工作者在该遗址 的唐代早期文化层下共发现隋代灰坑 6 座, 其中在 4 个灰坑中出土有透影白瓷, 可辨认的器型有碗、杯、 盘、砚、孟、器盖等. 从灰坑中共存器物都是典型的 隋代器物, 如高足盘、双系直口罐、玫口钵、长颈瓶、 针口瓶等, 推断这种透影白瓷应该为隋代产品. 在内 丘城关和临城祁村遗址中发掘出土的唐代遗物非常 丰富, 时间上涵盖唐早期、中唐至晚唐五代, 为探讨 邢窑唐代白瓷的发展奠定了基础. 唐早期的遗物中
白瓷非常少见, 而黄釉瓷和黑釉瓷较多. 内丘城关和 临城祁村中唐地层都出土了大量细白瓷标本, 种类 多而且造型美观、实用，虽然达不到隋代透影白瓷的 薄胎透影, 但也堪称瓷中精品, 其产量之大, 在当时 可能已成为“天下无贵贱通用之物”. 内丘城关窑址 上还出土了一定量“盈”字款器物残片, 为传世“盈”字 款白瓷及西安唐代大明宫遗址出土的“盈”字款白瓷 的出处找到了答案. 邢窑金代及以后的窑址一般不 与五代以前的窑址共存，而且其遗物特征、装饰方 法及装饰风格更多的与定窑相同，应属定窑系的产 品 $^{[1 \sim 3]}$.

自邢窑被发现以来, 考古界和科技界相继开展 了一些研究工作, 在考古类型学和科技研究等方面 都取得了一定的成果, 对邢窑白瓷的分类以及组成 工艺特点都有了一定的认识 ${ }^{[4 \sim 10]}$, 其中陈尧成等人 ${ }^{[5]}$ 首次发现了邢窑隋代透影白瓷胎釉中高钾含量的特 征, 并通过显微观察证实胎中加入了较多量的钾长 石, 对邢窑精细白瓷的复仿制工作起到了一定的指

英文版发表信息： Lu X K, Li W D, Luo H J, et al. A scientific study on the Xing kiln of ancient China. Sci China Tech Sci, 2012, 55: 2902-2919, doi: 10.1007/s11431-012-4960-0 
导性作用. 但是, 以前的科技研究针对邢窑隋唐遗物 开展较多, 而对五代之后的研究几乎为空白.

为进一步系统地探明邢窑白瓷的发展脉络, 本 研究对河北省文物研究所提供的内丘、临城等邢窑遗 址出土的 470 余件瓷器标本的化学组成、釉面白度、 烧成温度以及物理性能进行了系统的测试分析, 其 中化学组成测试采用美国 EDAX 公司产 EAGLE-III 型能量色散 $X$ 射线荧光分析仪, 烧成温度测试采用 德国 NETZSCH 公司产 DIL 402C 型热膨胀分析仪, 白度测试采用日本 MINOLTA 公司产 CMD700 型色 彩分析仪. 运用多元统计的分析方 法 $^{[11]}$ 对测试数据 进行了分析处理, 研究结果首次较为全面地反映了 邢窑自隋代至金代制瓷工艺的发展过程. 另外, 在前 人研究的基础上, 还着重探讨了隋代透影白瓷的烧 制工艺、唐代粗白瓷和细白瓷的分类标准、邢窑金代 的制瓷工艺特点及其与隋唐时期的异同等问题, 取 得一些新的非常有意义的结果.

\section{1 隋代样品的测试分析}

\section{1 实验及结果}

\subsection{1 样品的选取}

所有样品均由河北省文物研究所提供, 共选择 测试样品 130 件, 均出土于内丘城关西关北窑址, 其 中隋代青瓷标本 30 件(实验室编号为 04-XXX, 出土 单位为 88NXT1(2)、隋代中粗白瓷 29 件(实验室编号
为 01-XXX, 出土单位为 $88 \mathrm{NXT} 2 \mathrm{H} 4)$ 、隋代细白瓷 11 件(实验室编号为 $02-\mathrm{XXX}$, 出土单位为 $88 \mathrm{NXT} 2 \mathrm{H} 6$ )、 隋代透影白瓷 60 件(实验室编号为 03-XXX, 出土单 位为 $88 \mathrm{NXT} 2 \mathrm{H} 4)$, 部分标本照片见图 1.

\subsection{2 测试结果}

样品胎釉的化学组成测试结果见表 1 和 2 . 限于 文章篇幅, 表 1 中只列出了隋代青瓷、中粗白瓷和透 影白瓷胎釉的主次量元素氧化物含量的平均值和标 准差. 由于 11 件隋代细白瓷样品的组成波动性较大, 所以表 2 中将它们的胎釉组成全部列出.

\section{2 分析与讨论}

\subsection{1 样品的原料特点及分类}

图 2 和 3 是隋代样品胎和釉中 8 种主次量化学组 成 $\left(\mathrm{Na}_{2} \mathrm{O}, \mathrm{MgO}, \mathrm{Al}_{2} \mathrm{O}_{3}, \mathrm{SiO}_{2}, \mathrm{~K}_{2} \mathrm{O}, \mathrm{CaO}, \mathrm{TiO}_{2}, \mathrm{Fe}_{2} \mathrm{O}_{3}\right)$ 的二维对应分析分析结果.

从胎和釉的统计分析结果可以看出, 透影白瓷 的原料特征非常明显, 胎和釉中 $\mathrm{K}_{2} \mathrm{O}$ 含量都较高, 而 着色元素 $\mathrm{Fe}_{2} \mathrm{O}_{3}, \mathrm{TiO}_{2}$ 都极低. 透影白瓷的高钾釉是 我国最早出现的碱钻釉, 而在南方地区则迟至 700 余 年后的景德镇元代才开始出现 ${ }^{[12]}$. 部分细白瓷中的 胎、釉原料都与透影白瓷一致; 另一部分细白瓷胎中 $\mathrm{Al}_{2} \mathrm{O}_{3}$ 含量非常高, 而釉的组成处于中粗白瓷和透影 白瓷的过渡阶段. 中粗白瓷中有 2 个样品较为特殊 (01-033 和 034), 它们的胎、釉都较接近于胎中 $\mathrm{Al}_{2} \mathrm{O}_{3}$
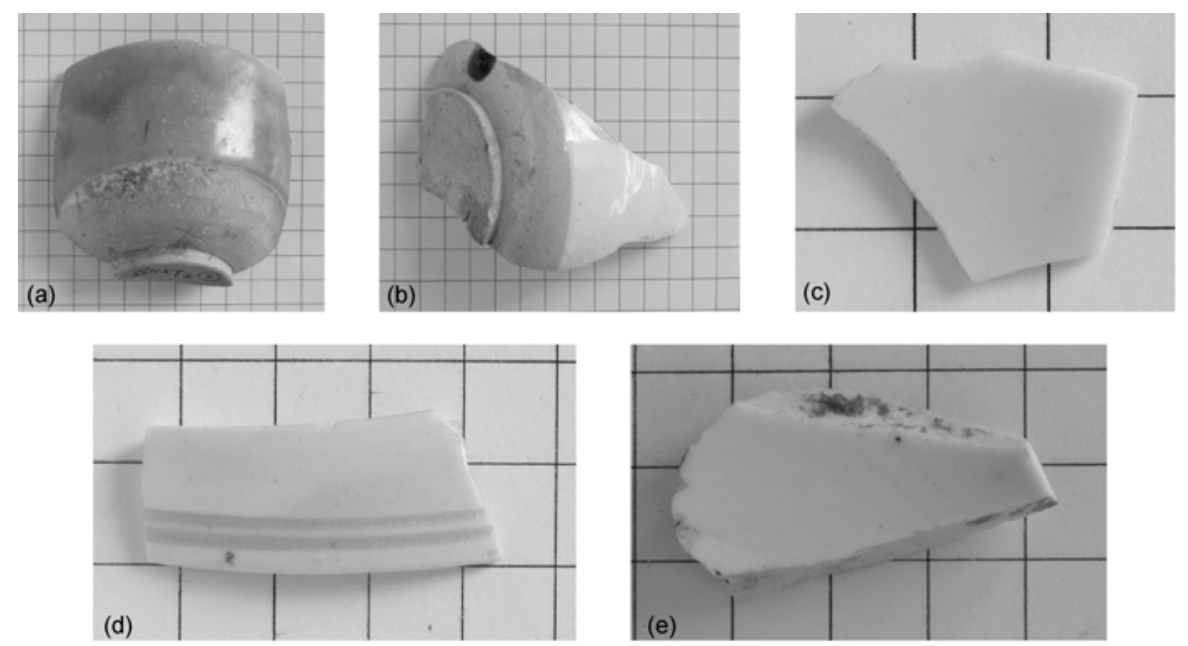

图 1 实验所选邢窑内丘西关北窑址出土隋代部分标本外观照片

(a) 青瓷 04-023; (b) 中粗白瓷 01-001; (c) 透影白瓷 03-016; (d) 第一类细白瓷 02-009; (e) 第二类细白瓷 02-002 
表 1 内丘西关北窑址样品胎、釉的化学组成数据 $(\mathrm{wt} \%)$

\begin{tabular}{|c|c|c|c|c|c|c|c|c|c|c|c|c|}
\hline Sort & Body/glaze & & $\mathrm{Na}_{2} \mathrm{O}$ & $\mathrm{MgO}$ & $\mathrm{Al}_{2} \mathrm{O}_{3}$ & $\mathrm{SiO}_{2}$ & $\mathrm{~K}_{2} \mathrm{O}$ & $\mathrm{CaO}$ & $\mathrm{TiO}_{2}$ & $\mathrm{Fe}_{2} \mathrm{O}_{3}$ & $\mathrm{MnO}$ & $\mathrm{P}_{2} \mathrm{O}_{5}$ \\
\hline \multirow{2}{*}{ Celadon } & \multirow{2}{*}{ Body } & Mean & 0.34 & 0.79 & 26.23 & 66.13 & 1.87 & 1.03 & 0.64 & 1.96 & 0.01 & 0.02 \\
\hline & & S.D. & 0.22 & 0.15 & 1.47 & 1.53 & 0.24 & 0.28 & 0.06 & 0.17 & 0.00 & 0.01 \\
\hline $\begin{array}{l}\text { Coarse white } \\
\text { porcelain }\end{array}$ & Body & $\begin{array}{l}\text { Mean } \\
\text { S.D. }\end{array}$ & $\begin{array}{l}0.37 \\
0.18\end{array}$ & $\begin{array}{l}0.86 \\
0.38\end{array}$ & $\begin{array}{c}29.15 \\
2.54\end{array}$ & $\begin{array}{c}63.78 \\
2.16\end{array}$ & $\begin{array}{l}1.42 \\
0.32\end{array}$ & $\begin{array}{l}0.70 \\
0.19\end{array}$ & $\begin{array}{l}0.76 \\
0.16\end{array}$ & $\begin{array}{l}1.97 \\
0.32\end{array}$ & $\begin{array}{l}0.02 \\
0.01\end{array}$ & $\begin{array}{l}0.02 \\
0.01\end{array}$ \\
\hline \multirow{2}{*}{$\begin{array}{l}\text { Translucent white } \\
\text { porcelain }\end{array}$} & \multirow{2}{*}{ Body } & Mean & 1.36 & 0.21 & 25.63 & 62.41 & 7.76 & 0.70 & 0.14 & 0.80 & 0.01 & 0.00 \\
\hline & & S.D. & 0.23 & 0.12 & 1.01 & 0.87 & 0.60 & 0.23 & 0.03 & 0.03 & 0.00 & 0.00 \\
\hline \multirow{2}{*}{ Celadon } & \multirow{2}{*}{ Glaze } & Mean & 0.60 & 2.74 & 12.11 & 64.87 & 2.90 & 12.78 & 0.27 & 2.73 & 0.06 & 0.21 \\
\hline & & S.D. & 0.41 & 0.52 & 1.42 & 2.39 & 0.71 & 2.31 & 0.05 & 0.23 & 0.01 & 0.05 \\
\hline \multirow{2}{*}{$\begin{array}{l}\text { Coarse white } \\
\text { porcelain }\end{array}$} & \multirow{2}{*}{ Glaze } & Mean & 0.53 & 3.32 & 14.02 & 65.49 & 2.28 & 12.14 & 0.09 & 1.14 & 0.04 & 0.19 \\
\hline & & S.D. & 0.44 & 0.65 & 1.70 & 2.56 & 0.73 & 3.07 & 0.05 & 0.27 & 0.01 & 0.05 \\
\hline \multirow{2}{*}{$\begin{array}{l}\text { Translucent white } \\
\text { porcelain }\end{array}$} & \multirow{2}{*}{ Glaze } & Mean & 1.21 & 2.73 & 13.58 & 70.60 & 6.54 & 3.41 & 0.05 & 0.89 & 0.03 & 0.06 \\
\hline & & S.D. & 0.54 & 0.99 & 1.26 & 1.65 & 0.81 & 1.45 & 0.02 & 0.07 & 0.01 & 0.02 \\
\hline
\end{tabular}

表 2 内丘西关北隋代细白瓷样品胎、釉的化学组成数据 (wt\%)

\begin{tabular}{|c|c|c|c|c|c|c|c|c|c|c|c|}
\hline Code No. & Body/glaze & $\mathrm{Na}_{2} \mathrm{O}$ & $\mathrm{MgO}$ & $\mathrm{Al}_{2} \mathrm{O}_{3}$ & $\mathrm{SiO}_{2}$ & $\mathrm{~K}_{2} \mathrm{O}$ & $\mathrm{CaO}$ & $\mathrm{TiO}_{2}$ & $\mathrm{Fe}_{2} \mathrm{O}_{3}$ & $\mathrm{MnO}$ & $\mathrm{P}_{2} \mathrm{O}_{5}$ \\
\hline $02-001-b$ & Body & 0.90 & 0.37 & 27.82 & 62.52 & 5.90 & 0.47 & 0.17 & 0.85 & 0.01 & 0.00 \\
\hline $02-002-b$ & Body & 1.18 & 0.38 & 27.83 & 62.07 & 6.05 & 0.48 & 0.17 & 0.84 & 0.01 & 0.00 \\
\hline $02-003-b$ & Body & 0.97 & 0.33 & 29.26 & 61.25 & 5.72 & 0.47 & 0.15 & 0.85 & 0.01 & 0.00 \\
\hline 02-004-b & Body & 1.54 & 0.25 & 27.04 & 60.88 & 7.93 & 0.40 & 0.14 & 0.83 & 0.00 & 0.00 \\
\hline $02-005-b$ & Body & 1.30 & 0.14 & 27.97 & 61.15 & 7.19 & 0.35 & 0.15 & 0.75 & 0.01 & 0.00 \\
\hline $02-006-b$ & Body & 1.12 & 0.39 & 28.68 & 61.18 & 6.16 & 0.49 & 0.14 & 0.84 & 0.01 & 0.00 \\
\hline 02-007-b & Body & 0.95 & 0.30 & 27.98 & 61.98 & 6.27 & 0.50 & 0.15 & 0.87 & 0.01 & 0.00 \\
\hline $02-008-b$ & Body & 0.29 & 1.49 & 32.51 & 61.80 & 0.27 & 1.25 & 0.28 & 1.11 & 0.01 & 0.00 \\
\hline $02-009-b$ & Body & 0.40 & 0.62 & 38.97 & 56.08 & 0.87 & 0.30 & 0.59 & 1.17 & 0.01 & 0.01 \\
\hline $02-010-b$ & Body & 0.22 & 0.60 & 37.83 & 57.53 & 0.79 & 0.34 & 0.52 & 1.17 & 0.01 & 0.01 \\
\hline $02-011-b$ & Body & 0.31 & 0.68 & 35.84 & 58.86 & 1.11 & 0.58 & 0.41 & 1.22 & 0.01 & 0.00 \\
\hline 02-001-g & Glaze & 0.33 & 2.75 & 14.55 & 71.93 & 5.58 & 2.83 & 0.05 & 0.96 & 0.02 & 0.06 \\
\hline $02-002-\mathrm{g}$ & Glaze & 0.16 & 3.03 & 13.37 & 72.32 & 5.43 & 3.58 & 0.04 & 1.06 & 0.03 & 0.07 \\
\hline $02-003-\mathrm{g}$ & Glaze & 1.75 & 4.37 & 13.21 & 68.47 & 5.66 & 4.45 & 0.06 & 1.03 & 0.03 & 0.09 \\
\hline $02-004-\mathrm{g}$ & Glaze & 0.88 & 3.21 & 13.66 & 70.62 & 6.04 & 3.73 & 0.05 & 0.81 & 0.03 & 0.07 \\
\hline $02-005-g$ & Glaze & 1.32 & 1.41 & 13.96 & 73.03 & 6.72 & 1.55 & 0.06 & 0.94 & 0.02 & 0.03 \\
\hline 02-006-g & Glaze & 1.53 & 2.47 & 15.31 & 70.52 & 5.84 & 2.39 & 0.06 & 0.88 & 0.02 & 0.05 \\
\hline $02-007-\mathrm{g}$ & Glaze & 1.80 & 2.77 & 15.57 & 69.53 & 5.95 & 2.43 & 0.05 & 0.91 & 0.02 & 0.06 \\
\hline 02-008-g & Glaze & 1.25 & 3.40 & 18.14 & 68.40 & 0.64 & 6.20 & 0.07 & 0.89 & 0.03 & 0.13 \\
\hline 02-009-g & Glaze & 0.91 & 2.60 & 15.33 & 69.55 & 2.54 & 6.77 & 0.08 & 1.23 & 0.05 & 0.14 \\
\hline 02-010-g & Glaze & 1.05 & 2.69 & 15.30 & 68.65 & 2.78 & 7.30 & 0.09 & 1.13 & 0.05 & 0.12 \\
\hline $02-011-\mathrm{g}$ & Glaze & 0.61 & 2.56 & 16.16 & 61.26 & 6.37 & 10.57 & 0.13 & 1.35 & 0.06 & 0.14 \\
\hline
\end{tabular}

含量高的那类细白瓷. 将多元统计分析中影响样品 分类的权重较大的因子组成作单元素散布分析, 如 图 4 和 5 所示.

从表 1 的数据和以上单元素的分析结果可以看 出, 隋代青瓷和隋代中粗白瓷的胎差别不是很大, 中 粗白瓷胎中 $\mathrm{Al}_{2} \mathrm{O}_{3}$ 含量略高于青瓷胎, 而着色元素 $\mathrm{TiO}_{2}$ 的含量比青瓷胎甚至还要略高一些. 青瓷釉和 中粗白瓷釉中 $\mathrm{Na}_{2} \mathrm{O}, \mathrm{MgO}, \mathrm{K}_{2} \mathrm{O}, \mathrm{CaO}$ 等熔剂含量也 基本相当, 主要差别在于中粗白瓷釉中着色元素
$\mathrm{Fe}_{2} \mathrm{O}_{3}(1.14 \%), \mathrm{TiO}_{2}(0.09 \%)$ 的含量比青瓷釉中 $\mathrm{Fe}_{2} \mathrm{O}_{3}(2.73 \%), \mathrm{TiO}_{2}(0.26 \%)$ 的含量明显降低. 另外, 青瓷和中粗白瓷的胎体较为粗粘, 都施加了白色化 妆土, 白瓷釉是透明高钙釉, 中粗白瓷釉中着色元素 铁和钛含量的降低使得化妆土的白色被直接反映出 来, 因此呈现了白瓷的基本特征.

隋代细白瓷和透影白瓷都不施加化妆土. 细白 瓷共分析样品 11 件, 从表 2 的数据以及图 2 和 3 的 统计分析结果, 可以将其划分为 2 类, 第一类(4 件, 


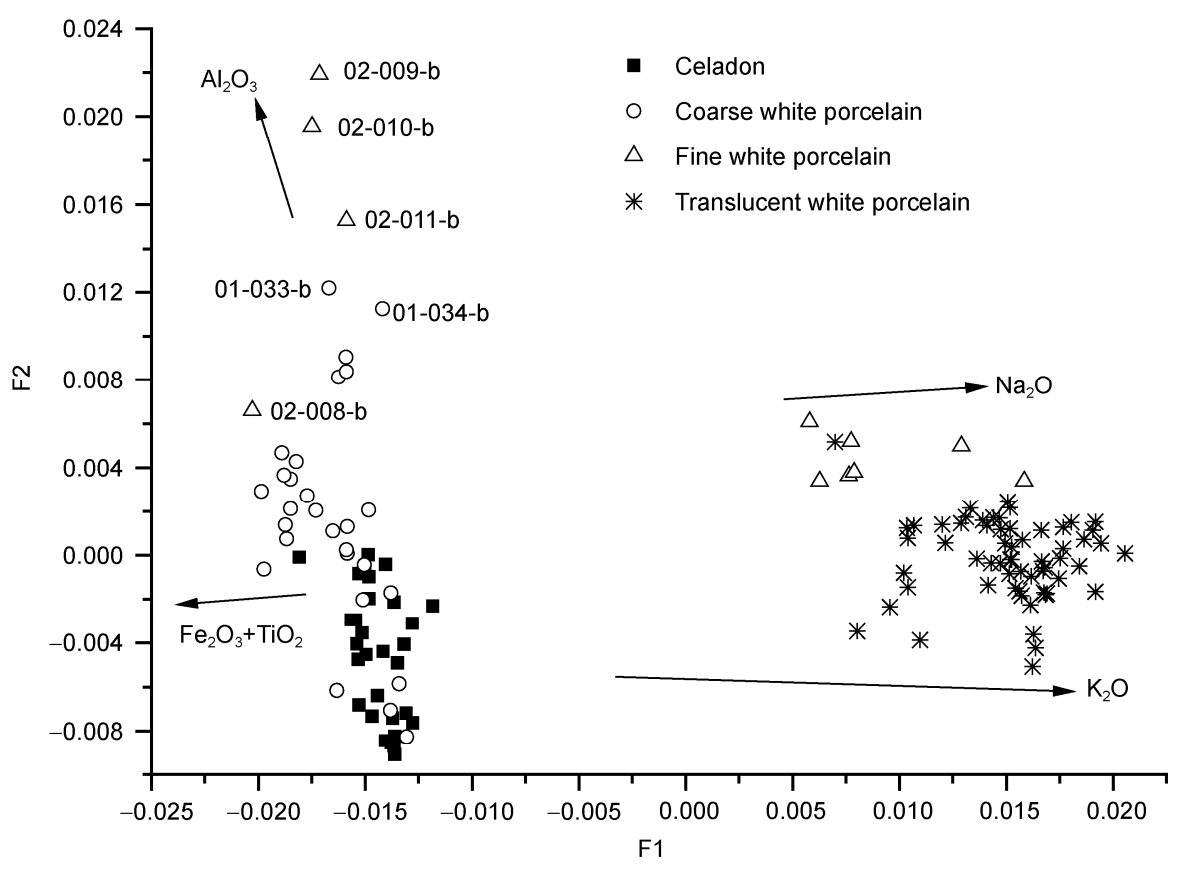

图 2 邢窑内丘西关北窑址隋代瓷胎的二维对应分析结果

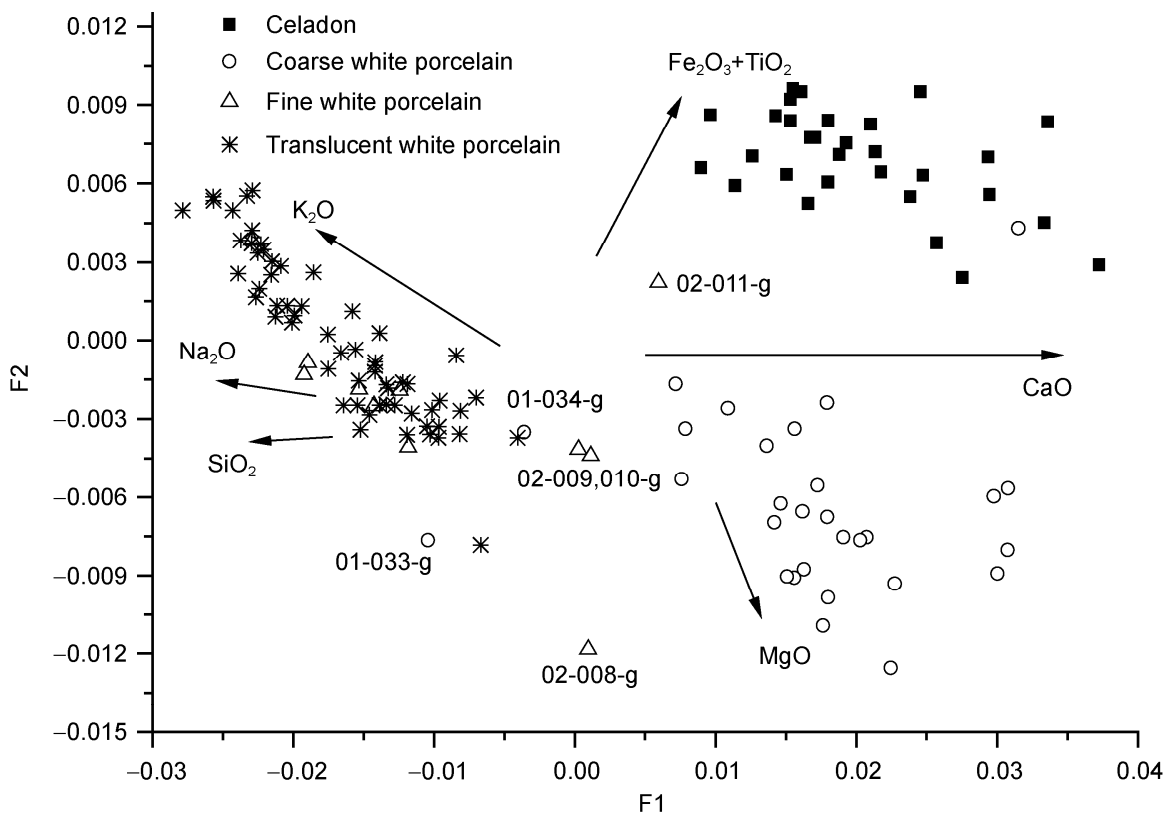

图 3 邢窑内丘西关北窑址隋代瓷釉的二维对应分析结果

02-008 至 011)胎中 $\mathrm{Al}_{2} \mathrm{O}_{3}$ 含量非常高, 而釉的组成处 于中粗白瓷和透影白瓷的过渡阶段; 第二类 $(7$ 件, 02-001 至 007)已经基本具备了透影白瓷的胎釉原料 特征. 这 2 种截然不同原料特征的细白瓷样品为我们 探讨透影白瓷的形成过程提供了绝佳的依据.
第一类细白瓷样品, 其胎中 $\mathrm{Al}_{2} \mathrm{O}_{3}$ 含量(均值 $36.29 \%$ )很高, 着色元素铁、钛含量相比中粗白瓷有 所降低. 它们釉料的组成又可以分为 2 种情况, 其一, 02-008 至 010 三个样品, 其 $\mathrm{CaO}$ 含量(6.76\%)明显低 于中粗白瓷 $(12.1 \%), \mathrm{K}_{2} \mathrm{O}$ 含量 $(1.99 \%)$ 与中粗白瓷 

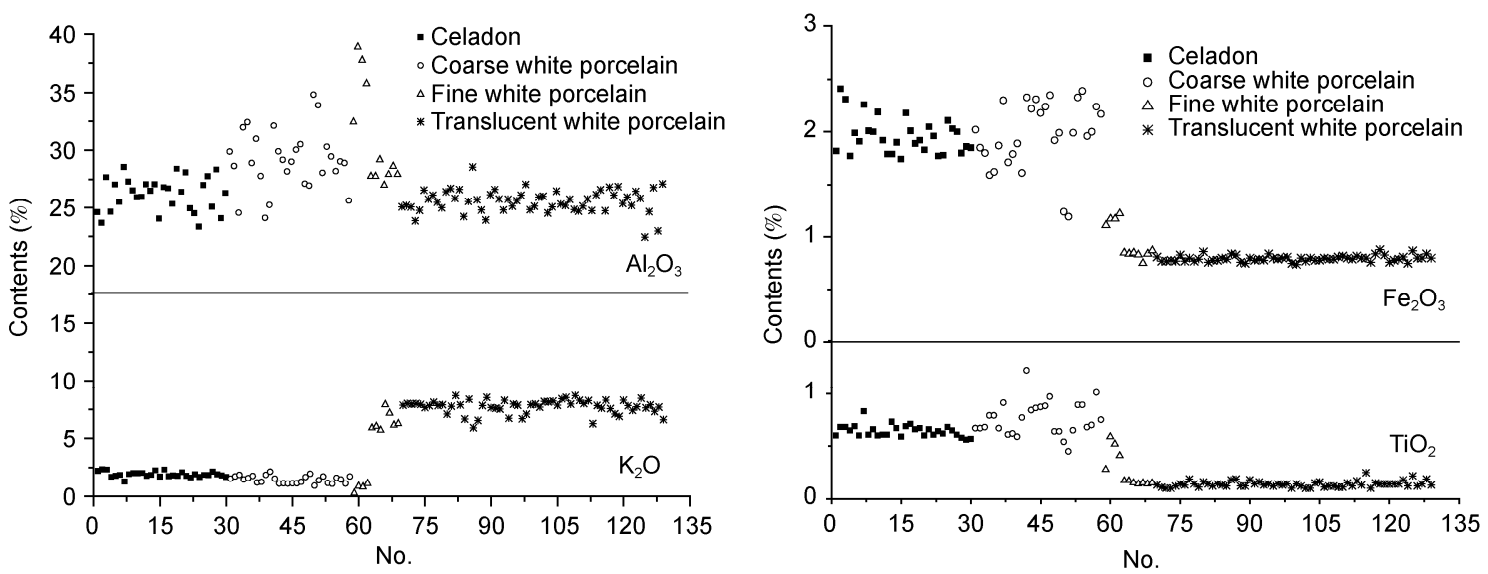

图 4 邢窑内丘隋代瓷胎中 $\mathrm{Al}_{2} \mathrm{O}_{3}, \mathrm{~K}_{2} \mathrm{O}, \mathrm{Fe}_{2} \mathrm{O}_{3}, \mathrm{TiO}_{2}$ 的分析结果
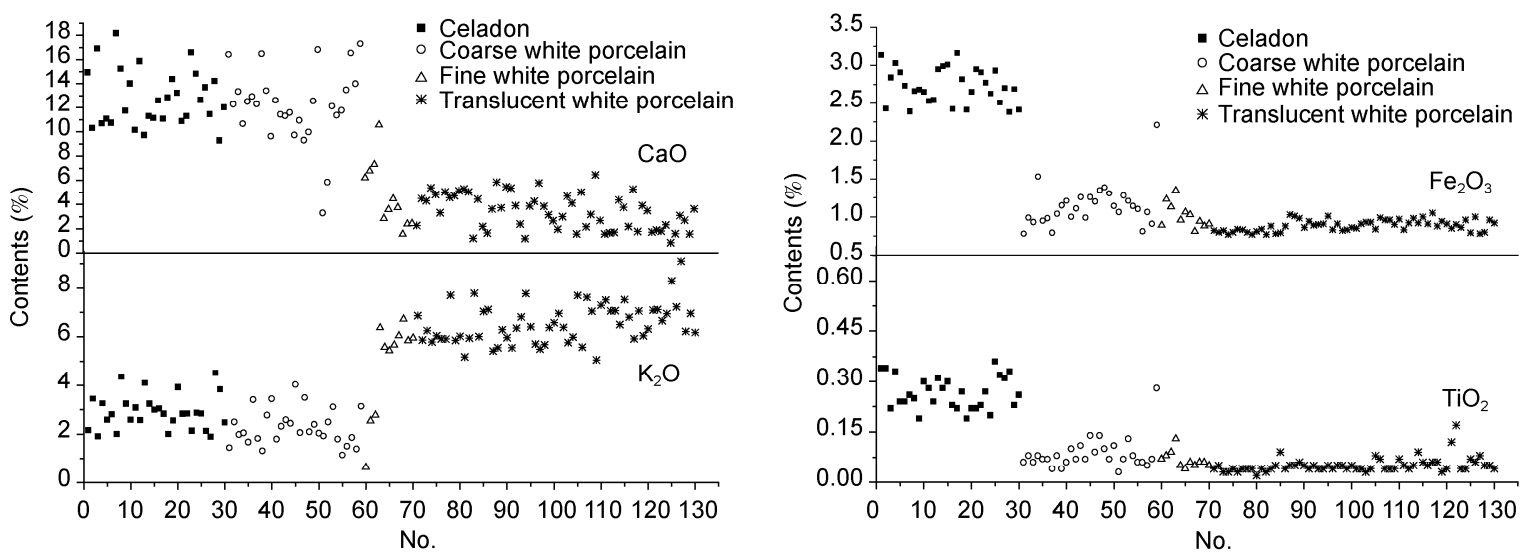

图 5 邢窑内丘隋代瓷釉中 $\mathrm{CaO}, \mathrm{K}_{2} \mathrm{O}, \mathrm{Fe}_{2} \mathrm{O}_{3}, \mathrm{TiO}_{2}$ 的分析结果

(2.28\%)相近. 我国古代瓷釉自原始瓷釉到越窑青瓷 釉, 基本都是以 $\mathrm{CaO}$ 为主要助熔剂, 而 $\mathrm{CaO}$ 的来源 一般是石灰石和草木灰 ${ }^{[12]}$, 以上的现象说明这 3 个细 白瓷的釉的配方有可能只是在中粗白瓷釉料基础上 大量减少了釉灰的使用量. 其二, 样品 02-011, 它的 釉中 $\mathrm{K}_{2} \mathrm{O}$ 含量已经增至 $6.37 \%$, 而 $\mathrm{CaO}$ 含量还较高 $(10.57 \%)$, 与中粗白瓷釉中 $\mathrm{CaO}$ 含量差别不大, 说明 这个样品的釉可能是在中粗白瓷釉的基础上引入了 高钾原料, 而釉灰的使用量变化不是很大. 高铝胎体 细白瓷釉的这 2 种情形, 体现了隋代陶工当时对釉料 配方的探索过程, 他们在同一种细白瓷胎的基础上 试验了 2 种不同的釉料工艺.

第二类细白瓷样品, 02-001 至 007, 这 7 个样品 胎和釉中 $\mathrm{K}_{2} \mathrm{O}$ 含量已分别增至 $6.46 \%$ 和 5.89\%, 已接 近透影白瓷胎釉中 $\mathrm{K}_{2} \mathrm{O}$ 含量水平, 而它们釉中 $\mathrm{CaO}$
的含量很低 $(2.99 \%)$. 前人研究发现透影白瓷胎中存 在钾长石残骸 ${ }^{[5]}$, 因此这些样品胎中 $\mathrm{K}_{2} \mathrm{O}$ 含量的大幅 提高, 应该同样与引入高钾原料有关. 统计分析图 3 指出, 第一类细白瓷釉处于中粗白瓷和透影白瓷的 过渡阶段, 因此第二类细白瓷样品有可能是以第一 类细白瓷样品为基础, 在胎和釉中都大量引入了高 钾原料的结果. 这类样品外观上胎体还较厚, 与透影 白瓷的薄胎相比有差别.

第二类细白瓷实际上已经具备了透影白瓷的原 料特征, 其厚度一般为 $3 \sim 6 \mathrm{~mm}$, 而透影白瓷厚度大 多为 1 2 mm, 所以透影白瓷的制作应该是古代陶工 对器物的胎体进行了刻意的减薄加工, 薄胎的透影 白瓷大多是碗、杯等器物, 因此它们胎体厚度的差别 也有可能对应于不同的器型功用. 透影白瓷由于胎 釉中 $\mathrm{K}_{2} \mathrm{O}$ 含量较高, 使得胎体玻璃相增多, 透明度增 
加, 再加上胎体较薄, 从而产生了透光的效果.

\subsection{2 透影白瓷及细白瓷的配方工艺}

关于透影白瓷的配方工艺，陈尧成等人 ${ }^{[5]}$ 已经做 过讨论, 其以赞皇白家窑白坩土和内丘神头长石为 原料, 采用 $6: 4$ 的配比作为透影白瓷胎的配方. 本文 前面的研究指出, 第一类细白瓷有可能是第二类细 白瓷形成的物质基础, 而考古工作者在内丘西关北 窑址的探方中还发现了陆烧过的长石碎块 ${ }^{[3]}$, 因此本 文以第一类细白瓷胎和釉(不包含 02-011 釉)为基础, 对内丘城关西关北窑址探方中出土的煅烧长石的添 加量再做配比计算, 胎配方如表 3 所示, 釉配方如表 4 所示.

从表 3 和 4 的结果可以看出, 将第一类细白瓷的 胎和釉与西关北探方出土的煅烧长石等比例混合, 与第二类细白瓷和透影白瓷相比, 所得胎配方基本 吻合; 所得釉配方 $\mathrm{Al}_{2} \mathrm{O}_{3}, \mathrm{~K}_{2} \mathrm{O}$ 含量偏高, $\mathrm{SiO}_{2}$ 含量明
显偏低. 从表 4 的数据可以看出, 第一类细白瓷釉以 及西关北探方出土的煅烧长石中 $\mathrm{SiO}_{2}$ 的含量都不超 过 $69 \%$, 而第二类细白瓷以及透影白瓷中 $\mathrm{SiO}_{2}$ 的含 量都接近 $71 \%$, 所以无论怎么设置配方, 所得釉中 $\mathrm{SiO}_{2}$ 的含量都会比第二类细白瓷和透影白瓷偏低较 多. 考古工作者在内丘城关西关北探方中除了发现 煅烧过的长石, 还发现了石英碎块, 因此在第二类细 白瓷和透影白瓷釉的配方中有可能还添加了石英 ${ }^{[3]}$. 我们以第一类细白瓷釉、西关北探方出土的煅烧长石 以及石英为基础, 再进行配比计算如表 5 所示, 当配 方为 5:4:1 的时候, 基本与第二类细白瓷和透影白瓷 釉的组成相近. 对于胎的配方, 虽然 5:5 和 6:4 的配比 结果相差不多, 但是在当时的时代环境下, 等比例混 合显然更容易操作. 其实, 釉的配方也体现了古代陶 工等比例混合的思想, 不同的是他们将 5 份长石中的 一份换成了石英.

前面已经提到, 隋代中粗白瓷中有 2 个样品

\section{表 3 胎料配方的计算 $(\mathrm{wt} \%)$}

\begin{tabular}{ccccccccc}
\hline Body recipe & $\mathrm{Na}_{2} \mathrm{O}$ & $\mathrm{MgO}$ & $\mathrm{Al}_{2} \mathrm{O}_{3}$ & $\mathrm{SiO}_{2}$ & $\mathrm{~K}_{2} \mathrm{O}$ & $\mathrm{CaO}$ & $\mathrm{TiO}_{2}$ & $\mathrm{Fe}_{2} \mathrm{O}_{3}$ \\
\hline A: mean value of the first kind of fine white porcelain & 0.31 & 0.85 & 36.29 & 58.57 & 0.76 & 0.62 & 0.45 & 1.17 \\
bodies & 1.12 & - & 17.92 & 66.21 & 14.30 & 1.03 & - & 0.15 \\
C: the calcined feldspar from Xiguanbei site & 0.72 & 0.43 & 27.11 & 62.39 & 7.53 & 0.83 & 0.23 & 0.66 \\
0.5A+0.5C & 0.63 & 0.51 & 28.94 & 61.63 & 6.18 & 0.78 & 0.27 & 0.76 \\
0.6A+0.4C & 1.14 & 0.31 & 28.08 & 61.57 & 6.46 & 0.45 & 0.15 & 0.83 \\
Mean value of the second kind fine of white porcelain \\
bodies
\end{tabular}

\section{表 4 釉料配方的计算 $(\mathrm{wt} \%)$}

\begin{tabular}{|c|c|c|c|c|c|c|c|c|c|}
\hline Glaze recipe & $\mathrm{Na}_{2} \mathrm{O}$ & $\mathrm{MgO}$ & $\mathrm{Al}_{2} \mathrm{O}_{3}$ & $\mathrm{SiO}_{2}$ & $\mathrm{~K}_{2} \mathrm{O}$ & $\mathrm{CaO}$ & $\mathrm{TiO}_{2}$ & $\mathrm{Fe}_{2} \mathrm{O}_{3}$ & $\mathrm{P}_{2} \mathrm{O}_{5}$ \\
\hline $\begin{array}{l}\text { B: mean value of the first kind of fine white porcelain } \\
\text { glazes }\end{array}$ & 1.07 & 2.90 & 16.26 & 68.87 & 1.99 & 6.76 & 0.08 & 1.08 & 0.13 \\
\hline C: the calcined feldspar from Xiguanbei site & 1.12 & - & 17.92 & 66.21 & 14.30 & 1.03 & - & 0.15 & \\
\hline $0.5 \mathrm{~B}+0.5 \mathrm{C}$ & 1.10 & 1.45 & 17.09 & 67.54 & 8.15 & 3.90 & 0.04 & 0.62 & 0.06 \\
\hline $0.6 \mathrm{~B}+0.4 \mathrm{C}$ & 1.09 & 1.74 & 16.92 & 67.81 & 6.91 & 4.47 & 0.05 & 0.71 & 0.08 \\
\hline $\begin{array}{l}\text { Mean value of the second kind of fine white porcelain } \\
\text { glazes }\end{array}$ & 1.11 & 2.86 & 14.23 & 70.92 & 5.89 & 2.99 & 0.06 & 0.94 & 0.06 \\
\hline Mean value of the translucent white porcelain glazes & 1.21 & 2.73 & 13.58 & 70.60 & 6.54 & 3.41 & 0.05 & 0.89 & 0.06 \\
\hline
\end{tabular}

表 5 釉料配方的计算 $(\mathrm{wt} \%)$

\begin{tabular}{|c|c|c|c|c|c|c|c|c|c|}
\hline Glaze recipe & $\mathrm{Na}_{2} \mathrm{O}$ & $\mathrm{MgO}$ & $\mathrm{Al}_{2} \mathrm{O}_{3}$ & $\mathrm{SiO}_{2}$ & $\mathrm{~K}_{2} \mathrm{O}$ & $\mathrm{CaO}$ & $\mathrm{TiO}_{2}$ & $\mathrm{Fe}_{2} \mathrm{O}_{3}$ & $\mathrm{P}_{2} \mathrm{O}_{5}$ \\
\hline $\begin{array}{l}\text { B: mean value of the first kind of fine white porcelain } \\
\text { glazes }\end{array}$ & 1.07 & 2.90 & 16.26 & 68.87 & 1.99 & 6.76 & 0.08 & 1.08 & 0.13 \\
\hline C: the calcined feldspar from Xiguanbei site & 1.12 & - & 17.92 & 66.21 & 14.30 & 1.03 & - & 0.15 & \\
\hline D: quartz from Xingtai & & & 0.10 & 98.04 & & & 0.01 & 0.07 & \\
\hline $0.5 \mathrm{~B}+0.4 \mathrm{C}+0.1 \mathrm{D}$ & 0.98 & 1.45 & 15.30 & 70.72 & 6.71 & 3.79 & 0.04 & 0.61 & 0.06 \\
\hline $\begin{array}{c}\text { Mean value of the second kind of fine white porcelain } \\
\text { glazes }\end{array}$ & 1.11 & 2.86 & 14.23 & 70.92 & 5.89 & 2.99 & 0.06 & 0.94 & 0.06 \\
\hline Mean value of translucent white porcelain glazes & 1.21 & 2.73 & 13.58 & 70.60 & 6.54 & 3.41 & 0.05 & 0.89 & 0.06 \\
\hline
\end{tabular}


(01-033 和 034), 它们与其他中粗白瓷胎釉原料相差 较大, 而与第一类细白瓷较为接近, 胎中 $\mathrm{Al}_{2} \mathrm{O}_{3}$ 含量 很高, 釉中 $\mathrm{CaO}$ 含量很低. 它们胎体细淢, 颜色较白, 并且不施化妆土, 因此这两件样品其实应该归为细 白瓷之列. 对于这些高铝质胎体细白瓷的成因, 从对 中粗白瓷上化妆土的测试数据中可以找到一些依据.

从表 6 的数据可以看出, 这类细白瓷的胎体组成 与化妆土的组成比较接近, 因此推测这种细白瓷有 可能使用了与化妆土相似的, 质量较好、白度较高、 较纯净的高岭土做胎, 并不是在中粗白瓷胎的基础 上进行的改良. 另外, 从中粗白瓷釉到第一类细白瓷 釉, 由于其中 $\mathrm{CaO}$ 含量由 $12.1 \%$ 降至 $6.76 \%$, 而 $\mathrm{P}_{2} \mathrm{O}_{5}$ 含量由 $0.2 \%$ 降至 $0.13 \%$, 推测第一类细白瓷釉灰的 使用量可能在中粗白瓷釉的基础上大致减少了 $50 \%$ 左右.

\subsection{3 烧成制度与陶瓷性能}

从烧成温度的测试结果来看, 隋代青瓷烧成温 度均值为 $1250^{\circ} \mathrm{C}$, 隋代中粗白瓷烧成温度均值为 $1270^{\circ} \mathrm{C}$. 细白瓷和透影白瓷受样品尺寸限制, 所测样 品数量不是很多, 第一类高铝胎体细白瓷的烧成温 度较高为 $1350^{\circ} \mathrm{C}\left(3\right.$ 个样品 $\left.1340^{\circ} \mathrm{C}, 1350^{\circ} \mathrm{C}, 1360^{\circ} \mathrm{C}\right)$, 第二类细白瓷烧成温度 $1320^{\circ} \mathrm{C}$, 而透影白瓷的烧成 温度 $1260^{\circ} \mathrm{C}\left(3\right.$ 个样品 $\left.1230^{\circ} \mathrm{C}, 1250^{\circ} \mathrm{C}, 1290^{\circ} \mathrm{C}\right)$. 另外, 考古工作者指出根据窑址中出土瓷片与窑具的粘连 情况，推测隋代青瓷、中粗白瓷、透影白瓷可能为同 窑烧制, 而我们目前的实验测量数据也初步表明它 们的烧成温度较为相近. 第一类细白瓷烧成温度较 高, 而其釉的配方与中粗白瓷的最大区别在于 $\mathrm{CaO}$ 含量明显降低, 这是因为高铝胎体的完全烧结需要 更高的窑炉温度, 如不减少釉中熔剂则会造成严重 的流釉现象; 到了第二类细白瓷或透影白瓷, 随着胎 中加入长石, 胎中熔剂含量增加, 则又不需要那么高 的烧成温度. 烧成温度与原料组成之间对应关系的 变化, 也说明邢窑隋代陶工当时已经掌握了相当成 熟的制瓷技艺. 另外, 隋代青瓷的色调普遍泛黄, 又 与白瓷同窑烧制, 所以它们应该都是在偏氧化的气 氛下烧成的, 而北方烧制白瓷也多用氧化焰. 从胎体
吸水率的测试结果来看, 隋代青瓷和中粗白瓷吸水 率基本相当, 均值为 $4.1 \%$, 这与它们的烧成温度和 原料组成都较接近的情况也相对应; 透影白瓷吸水 率则非常低, 均值为 $0.5 \%$, 可见胎体已经非常致密; 细白瓷样品由于受样品尺寸限制, 没有做相关测试.

图 7 的色度测试结果表明, 隋代中粗白瓷、第一 类细白瓷、第二类细白瓷以及透影白瓷釉面 Hunter 白度均值依次变化为: $72.39 \% \rightarrow 79.59 \% \rightarrow 80.93 \% \rightarrow$ $77.73 \%$. 中粗白瓷由于釉料中着色元素铁钛含量比 青瓷明显降低, 再加上化妆土的底色, 使得釉面白度 由青瓷的 $49.49 \%$ 提高到 $72.39 \%$, 跨入了白瓷的行列.

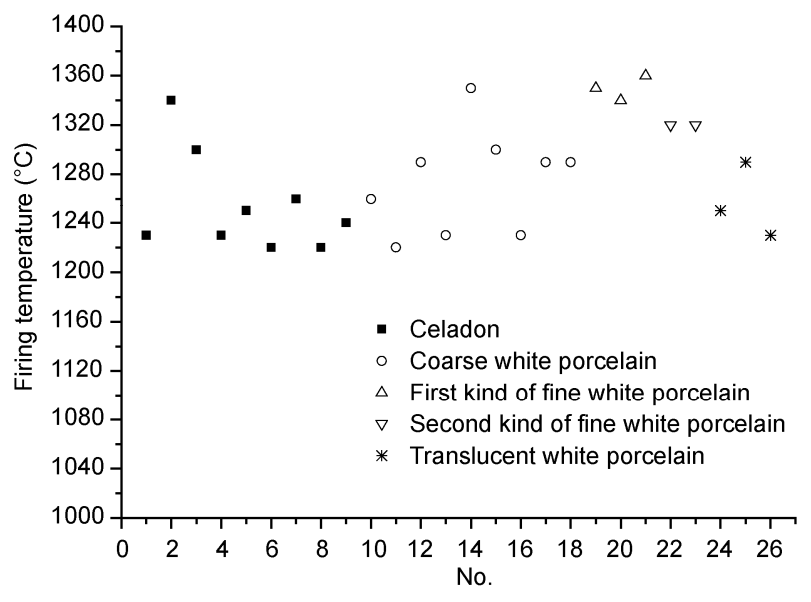

图 6 邢窑内丘西关北窑址隋代样品烧成温度

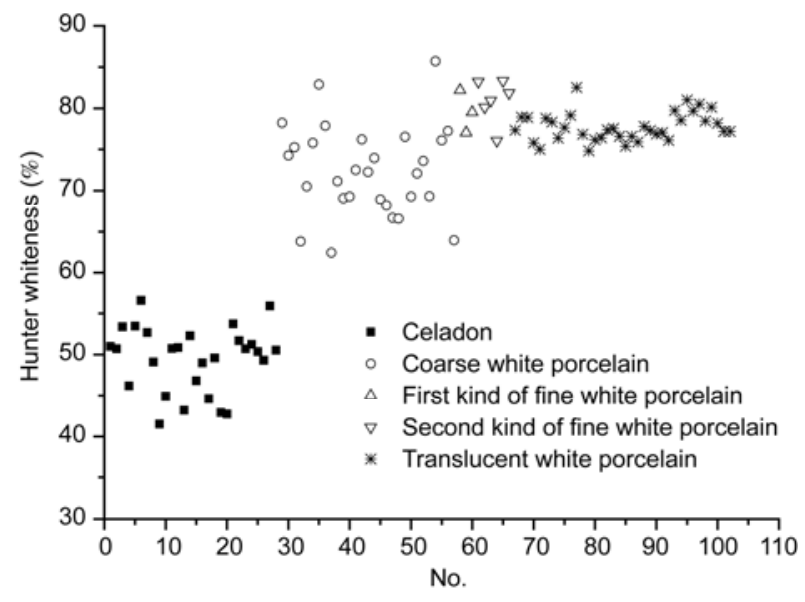

图 7 邢窑内丘西关北窑址隋代样品 Hunter 白度变化

\section{表 6 第一类细白瓷胎与化妆土组成的比较 $(w t \%)$}

\begin{tabular}{lcccccccc}
\hline & $\mathrm{Na}_{2} \mathrm{O}$ & $\mathrm{MgO}$ & $\mathrm{Al}_{2} \mathrm{O}_{3}$ & $\mathrm{SiO}_{2}$ & $\mathrm{~K}_{2} \mathrm{O}$ & $\mathrm{CaO}$ & $\mathrm{TiO}_{2}$ & $\mathrm{Fe}_{2} \mathrm{O}_{3}$ \\
\hline Mean value of the first kind of fine white porcelain bodies & 0.31 & 0.85 & 36.29 & 58.57 & 0.76 & 0.62 & 0.45 & 1.17 \\
Mean value of the engobes on the coarse white porcelain & 0.39 & 0.80 & 34.20 & 57.10 & 1.78 & 2.74 & 0.60 & 1.38 \\
\hline
\end{tabular}


透影白瓷与第二类细白瓷原料组成特点一致, 而白 度却有所降低, 是由于它们胎体透明度高而又较薄, 透光性较大造成的.

\section{2 唐代样品的测试分析}

\section{1 样品的选取}

所有样品及其相应的分期、分类信息均由河北省 文物研究所提供, 共选择测试样品 212 件, 具体信息 见表 7 .

\section{2 分析与讨论}

\subsection{1 样品的分类}

图 8 和 9 是样品胎和釉中 8 种主次量化学组成
$\left(\mathrm{Na}_{2} \mathrm{O}, \mathrm{MgO}, \mathrm{Al}_{2} \mathrm{O}_{3}, \mathrm{SiO}_{2}, \mathrm{~K}_{2} \mathrm{O}, \mathrm{CaO}, \mathrm{TiO}_{2}, \mathrm{Fe}_{2} \mathrm{O}_{3}\right)$ 的 二维对应分析分析结果.

从胎的统计分析结果可以看出，唐早期的黄釉 瓷和黑釉瓷胎料中 $\mathrm{Fe}_{2} \mathrm{O}_{3}$ 和 $\mathrm{TiO}_{2}$ 含量明显较高, 图 8 的椭圆形区域内包含了全部的黑釉瓷样品和大部分 黄釉瓷样品, 它们胎中铁含量都大于 $2.5 \%$; 中唐细 白瓷的胎料特征非常明显, $\mathrm{Al}_{2} \mathrm{O}_{3}$ 含量较高而着色元 素铁、钛的含量较低, 样品都分布于虚线以左, 只有 一个样品例外; 而中粗白瓷的胎料组成大部分介于 以上两者之间. 但是, 不可回避的是, 中粗白瓷中有 相当多的样品混在了细白瓷样品点之中, 具体情况 为: 高邑北焦盛唐至中唐中粗白瓷中 12 个(GY-BJ-01$001,002,005,007-015)$, 内丘城关和临城祁村中唐中 粗白瓷样品 9 个(NQ-CHG-09-005, 025, 047, 048, 049,

\section{表 7 邢窑唐代样品信息列表}

\begin{tabular}{|c|c|c|c|c|}
\hline Serial number & Period & Sort & Site & Sum \\
\hline NQ-CHG-07-XXX & Early Tang dynasty & Yellow porcelain & Neiqiu-Chengguan & 38 \\
\hline NQ-CHG-08-XXX & Early Tang dynasty & Black porcelain & Neiqiu-Chengguan & 20 \\
\hline GY-BJ-01-XXX & Prosperous Tang to middle Tang dynasty & Coarse white porcelain & Gaoyi-beijiao & 15 \\
\hline NQ-CHG-09-XXX & Middle Tang dynasty & Coarse white porcelain & Neiqiu-Chengguan & 35 \\
\hline NQ-CHG-10-XXX & Middle Tang dynasty & Fine white porcelain & Neiqiu-Chengguan & 32 \\
\hline LCH-QC-01-XXX & Middle Tang dynasty & Coarse white porcelain & Lincheng-Qicun & 7 \\
\hline LCH-QC-00-XXX & Middle Tang dynasty & Fine white porcelain & Lincheng-Qicun & 30 \\
\hline NQ-CHG-11-XXX & Late Tang-Five dynasties & Coarse white porcelain & Neiqiu-Chengguan & 4 \\
\hline LCH-QC-02-XXX & Late Tang-Five dynasties & Coarse white porcelain & Lincheng-Qicun & 31 \\
\hline
\end{tabular}

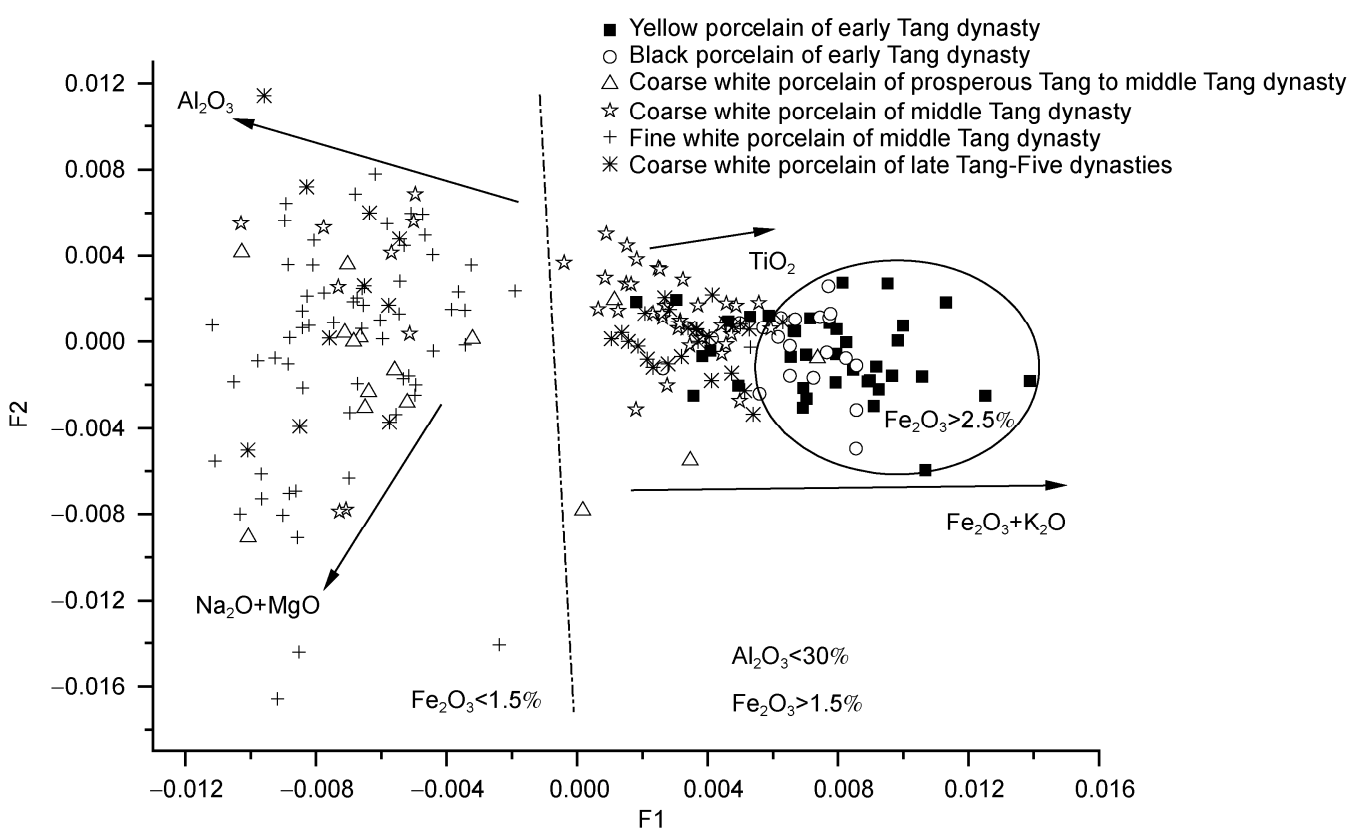

图 8 邢窑唐代瓷胎的二维对应分析结果 


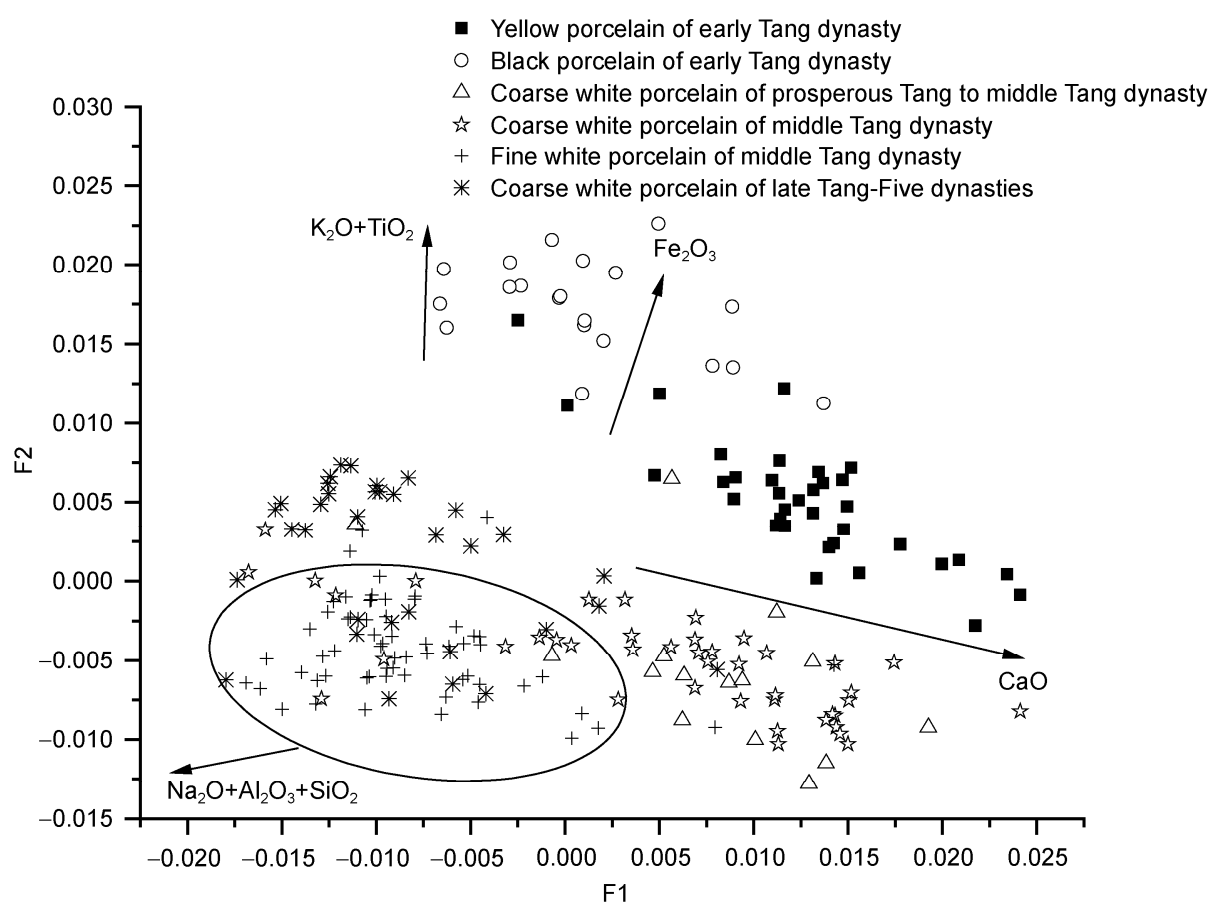

图 9 邢窑唐代瓷嬏的二维对应分析结果

052, 053; LCH-QC-01-001，002), 临城祁村晚唐五代 中粗白瓷样品 10 个(LQ-QC-02-001，002，004，014, $017,018,019,022,025,055)$. 这些样品与中唐的细白 瓷胎料组成较为接近, 它们具有明显的共性特征: 胎 体细淢、颜色较白、不施加化妆土、胎中 $\mathrm{Fe}_{2} \mathrm{O}_{3}$ 含量 低于 $1.5 \%$. 根据组成以及外观特征, 我们认为这些 样品实应归为细白瓷之列. 中唐细白瓷中的那个例 外样品(NQ-CHG-10-061)其实应归入中粗白瓷中, 它 同样施化妆土, 胎色较深.

唐早期黄嬏瓷所测试的样品中, 有 3 个样品的釉 是铅釉(NQ-CHG-07-026, 040, 047), 而其余都为钻釉, 由于它们属于不同的釉料体系, 化学组成数据差异 太大, 因此釉的统计分析中不包含这 3 个铅釉样品. 从釉的统计分析结果可以看出, 黄釉瓷及中唐之前 的中粗白瓷釉中 $\mathrm{CaO}$ 含量较高, 而细白瓷和晚唐五 代中粗白瓷釉中 $\mathrm{CaO}$ 含量相对较低, 黑釉瓷中 $\mathrm{Fe}_{2} \mathrm{O}_{3}$ 和 $\mathrm{TiO}_{2}$ 含量最高. 图 9 的椭圆形区域内包含了绝大 部分的中唐细白瓷, 还包括了通过对胎料分析, 中唐 中粗白瓷被认为是细白瓷的 9 个样品中的 7 个、晚唐 五代中粗白瓷中被认为是细白瓷的全部 10 个样品. 盛唐至中唐时期高邑北焦出土的中粗白瓷釉料则与 这些细白瓷不同, 虽然它们中大多数样品在胎料的
分析中被认为应该归为细白瓷之列。

诚然，釉料的统计结果不完全相同于胎料的分 析结果, 但是大部分还是相似的, 这也基本证明了我 们从胎的组成来划分中粗白瓷和细白瓷的正确性. 所以，细白瓷区别于中粗白瓷很重要的一个方面就 是胎料的选取，优质的胎料是制作精细白瓷的物质 基础. 唐代细白瓷由于胎料优质, 胎质细淢、胎色较 白, 不需要化妆土就能呈现白瓷的基本特征. 中粗白 瓷则由于胎质较粗, 着色元素铁钛含量较高, 而需要 依靠白色化妆土呈色. 因此从外观上, 是否施加化妆 土可以作为中粗白瓷和细白瓷的区分标准之一; 而 从技术角度上，对于邢窑唐代的中粗白瓷和细白瓷， 其胎中 $\mathrm{Fe}_{2} \mathrm{O}_{3}$ 含量大于或小于 $1.5 \%$ 可作为区分它们 的标准之一.

本部分有关邢窑唐代研究的以下图表及分析中 将采用新的分类名称，即将中唐中粗白瓷中的那 9 个 样品归入中唐细白瓷、将晚唐五代中粗白瓷中那 10 个样品单独列为晚唐五代细白瓷，有鉴于高邑北焦 盛唐至中唐白瓷釉的特殊性, 它的称谓仍然沿用中 粗白瓷.

\subsection{2 样品的原料特点及演变}

样品胎釉的化学组成测试结果见表 8 . 限于文章 
表 8 邢窑唐代样品胎、釉的主次量化学组成数据 (wt\%)

\begin{tabular}{|c|c|c|c|c|c|c|c|c|c|c|c|c|c|}
\hline Period & Sort & Body/glaze & & $\mathrm{Na}_{2} \mathrm{O}$ & $\mathrm{MgO}$ & $\mathrm{Al}_{2} \mathrm{O}_{3}$ & $\mathrm{SiO}_{2}$ & $\mathrm{~K}_{2} \mathrm{O}$ & $\mathrm{CaO}$ & $\mathrm{TiO}_{2}$ & $\mathrm{Fe}_{2} \mathrm{O}_{3}$ & $\mathrm{MnO}$ & $\mathrm{P}_{2} \mathrm{O}_{5}$ \\
\hline \multirow{2}{*}{ Early Tang dynasty } & \multirow{2}{*}{$\begin{array}{l}\text { Yellow } \\
\text { porcelain }\end{array}$} & \multirow{2}{*}{ Body } & Mean & 0.36 & 0.92 & 25.46 & 65.58 & 2.22 & 0.98 & 0.57 & 2.90 & 0.02 & 0.03 \\
\hline & & & S.D. & 0.18 & 0.11 & 1.55 & 1.17 & 0.27 & 0.24 & 0.04 & 0.37 & 0.01 & 0.01 \\
\hline \multirow{2}{*}{ Early Tang dynasty } & \multirow{2}{*}{$\begin{array}{c}\text { Black } \\
\text { porcelain }\end{array}$} & \multirow{2}{*}{ Body } & Mean & 0.35 & 0.89 & 25.76 & 65.69 & & 0.94 & 0.60 & 2.66 & 0.02 & 0.03 \\
\hline & & & S.D. & 0.19 & 0.14 & 1.03 & 0.99 & 0.15 & 0.21 & 0.03 & 0.39 & 0.00 & .01 \\
\hline \multirow{2}{*}{$\begin{array}{c}\text { Prosperous Tang to } \\
\text { middle Tang } \\
\text { dynasty }\end{array}$} & \multirow{2}{*}{$\begin{array}{l}\text { Coarse white } \\
\text { porcelain }\end{array}$} & \multirow{2}{*}{ Body } & Mean & 0.47 & 1.72 & 28.33 & 64.38 & 0.94 & 1.32 & 0.48 & 1.36 & 0.03 & 0.02 \\
\hline & & & S.D. & 0.32 & 0.48 & 2.78 & 2.69 & 0.50 & 0.47 & 0.16 & 0.34 & 0.02 & 0.01 \\
\hline \multirow{2}{*}{$\begin{array}{c}\text { Middle Tang } \\
\text { dynasty }\end{array}$} & \multirow{2}{*}{$\begin{array}{l}\text { Coarse white } \\
\text { porcelain }\end{array}$} & \multirow{2}{*}{ Body } & Mean & 0.34 & 0.88 & 27.85 & 64.31 & 1.80 & 1.01 & 0.64 & 2.17 & 0.02 & 0.03 \\
\hline & & & S.D. & 0.17 & 0.13 & 1.49 & 1.38 & 0.21 & 0.38 & 0.06 & 0.26 & 0.01 & 0.01 \\
\hline \multirow{2}{*}{$\begin{array}{l}\text { Middle Tang } \\
\text { dynasty }\end{array}$} & \multirow{2}{*}{$\begin{array}{l}\text { Fine white } \\
\text { porcelain }\end{array}$} & \multirow{2}{*}{ Body } & Mean & 0.65 & 1.42 & 30.97 & 62.37 & 0.95 & 1.20 & 0.34 & 1.10 & 0.02 & 0.01 \\
\hline & & & S.D. & 0.45 & 0.50 & 2.20 & 1.88 & 0.44 & 0.57 & 0.09 & 0.12 & 0.01 & 0.01 \\
\hline \multirow{2}{*}{$\begin{array}{l}\text { Late Tang-Five } \\
\text { dynasties }\end{array}$} & \multirow{2}{*}{$\begin{array}{c}\text { Coarse white } \\
\text { porcelain }\end{array}$} & \multirow{2}{*}{ Body } & Mean & 0.39 & 0.85 & 27.16 & 65.31 & 1.83 & 0.76 & 0.70 & 2.00 & 0.01 & 0.02 \\
\hline & & & S.D. & 0.13 & 0.10 & 1.19 & 1.20 & 0.19 & 0.19 & 0.07 & 0.25 & 0.00 & 0.01 \\
\hline \multirow{2}{*}{$\begin{array}{l}\text { Late Tang-Five } \\
\text { dynasties }\end{array}$} & \multirow{2}{*}{$\begin{array}{l}\text { Fine white } \\
\text { porcelain }\end{array}$} & \multirow{2}{*}{ Body } & Mean & 0.63 & 1.10 & 32.20 & 61.28 & 0.92 & 1.37 & 0.37 & 1.15 & 0.03 & 0.02 \\
\hline & & & S.D. & 0.41 & 0.38 & 2.03 & 2.09 & 0.32 & 0.61 & 0.09 & 0.07 & 0.01 & 0.01 \\
\hline \multirow{2}{*}{ Early Tang dynasty } & \multirow{2}{*}{$\begin{array}{c}\text { Yellow } \\
\text { porcelain }\end{array}$} & \multirow[b]{2}{*}{ Glaze } & Mean & 0.6 & 2.5 & 12.22 & 64.20 & 2.54 & 13.18 & 0.36 & 3.38 & 0.07 & 0.18 \\
\hline & & & S.D. & 0.37 & 0.41 & 1.37 & 2.63 & 0.90 & 2.53 & 0.05 & 0.42 & 0.02 & 0.05 \\
\hline \multirow{2}{*}{ Early Tang dynasty } & \multirow{2}{*}{$\begin{array}{c}\text { Black } \\
\text { porcelain }\end{array}$} & \multirow{2}{*}{ Glaze } & Mean & 0.79 & 3.48 & 12.02 & 66.46 & 3.81 & 7.27 & 0.38 & 4.79 & 0.07 & 0.08 \\
\hline & & & S.D. & 0.44 & 0.52 & 0.88 & 1.86 & 1.00 & 2.24 & 0.04 & 0.45 & 0.01 & 0.03 \\
\hline \multirow{2}{*}{$\begin{array}{l}\text { Prosperous Tang to } \\
\text { middle Tang } \\
\text { dynasty }\end{array}$} & Coars & & Mean & 0.5 & 4.3 & 15.61 & 62.98 & & 12.01 & 0.11 & 1.34 & 0.09 & 0.21 \\
\hline & porc & Glaze & S.D. & 0.43 & & 1.66 & 3.12 & & 3.19 & 0.06 & 0.20 & 0.03 & 0.06 \\
\hline Midd & ite & & Mean & 0.61 & 3.20 & 14.36 & 65.07 & 1.97 & 12.49 & 0.09 & 1.23 & 0.09 & 0.24 \\
\hline & & Glaze & S.D. & 0.52 & 0.79 & 1.23 & 2.74 & 0.71 & 3.61 & 0.05 & 0.32 & 0.03 & 0.09 \\
\hline Middle Tang & Fine white & & Mean & 1.26 & 2.89 & 16.23 & 69.46 & 1.50 & 6.41 & 0.07 & 1.18 & 0.06 & 0.13 \\
\hline dynasty & porcelain & aze & S.D. & 0.83 & 0.94 & 1.48 & 2.40 & 0.76 & 2.27 & 0.05 & 0.19 & 0.02 & 0.07 \\
\hline Late $\mathrm{Ta}$ & Coar & f & Mean & 0.60 & 5.05 & 15.60 & 68.33 & 2.80 & 4.72 & 0.25 & 1.65 & 0.03 & 0.13 \\
\hline & & Glaze & S.D. & 0.60 & 2.32 & 1.66 & 1.81 & 0.44 & 2.63 & 0.10 & 0.33 & 0.02 & 0.05 \\
\hline Late Tang-Five & Fine white & & Mean & 1.50 & 2.71 & 15.77 & 69.98 & 1.56 & 6.31 & 0.07 & 1.11 & 0.07 & 0.14 \\
\hline dynasties & porcelain & & S.D. & 0.79 & 0.87 & 1.17 & 1.19 & 0.61 & 1.59 & 0.03 & 0.23 & 0.02 & 0.05 \\
\hline
\end{tabular}

篇幅, 表 8 中只列出它们的平均值和标准差. 将多元 统计分析中影响样品分类的权重较大的因子组成作 单元素散布分析, 如图 10 和 11 所示.

在以上的图表分析中, 样品的分类只采用时代 和瓷种作为区分标志, 而同一时代、同一瓷种的样品 其实有不同的产地，比如中唐中粗白瓷、中唐细白瓷 以及晚唐五代中粗白瓷都来自于内丘城关和临城祁 村 2 个窑址. 图 8 至 11 的统计分析结果指出, 样品的 组成特点在时代和瓷种方面存在较为明显的演变规 律, 同一时代、同一瓷种样品的组成特征较为一致, 说明内丘城关和临城祁村的样品并不突显地区性 差异.

胎料的分析结果表明，唐早期的黄釉瓷和黑釉 瓷胎中 $\mathrm{Fe}_{2} \mathrm{O}_{3}$ 含量较高, 均值分别为 $2.90 \%$ 和 $2.66 \%$,
外观上看，它们胎质粗䊁、胎色较深、有些呈黑色，而 表面大多都施白色化妆土. 中唐中粗白瓷的胎料相 比黄釉瓷和黑釉瓷有所改进, $\mathrm{Fe}_{2} \mathrm{O}_{3}$ 含量有所降低 $(2.17 \%)$, 但胎体颜色仍然较深, 表面也都施加化妆 土. 中、晚唐细白瓷的胎料则与中粗白瓷、黄釉瓷和 黑釉瓷的胎料明显不同, 比如中唐细白瓷其 $\mathrm{Al}_{2} \mathrm{O}_{3}$ 含 量 $(30.97 \%)$ 较中唐中粗白瓷 $(27.85 \%)$ 为高, 而着色元 素铁、钛的含量 $(1.10 \%, 0.34 \%)$ 较中粗白瓷 $(2.17 \%$, $0.64 \%$ )低了一半左右, 晚唐五代细白瓷与中粗白瓷 的差别与中唐时期的情况一致. 细白瓷样品的胎色 较白, 不施加化妆土, 很显然是古代陶工专门为制作 这一类别的产品所选择的较为优质的原料. 高邑北 焦盛唐-中唐中粗白瓷胎中铁钛含量也较低, 但是其 胎中 $\mathrm{Al}_{2} \mathrm{O}_{3}$ 含量均值为 $28.33 \%$, 却低于中晚唐的细白 

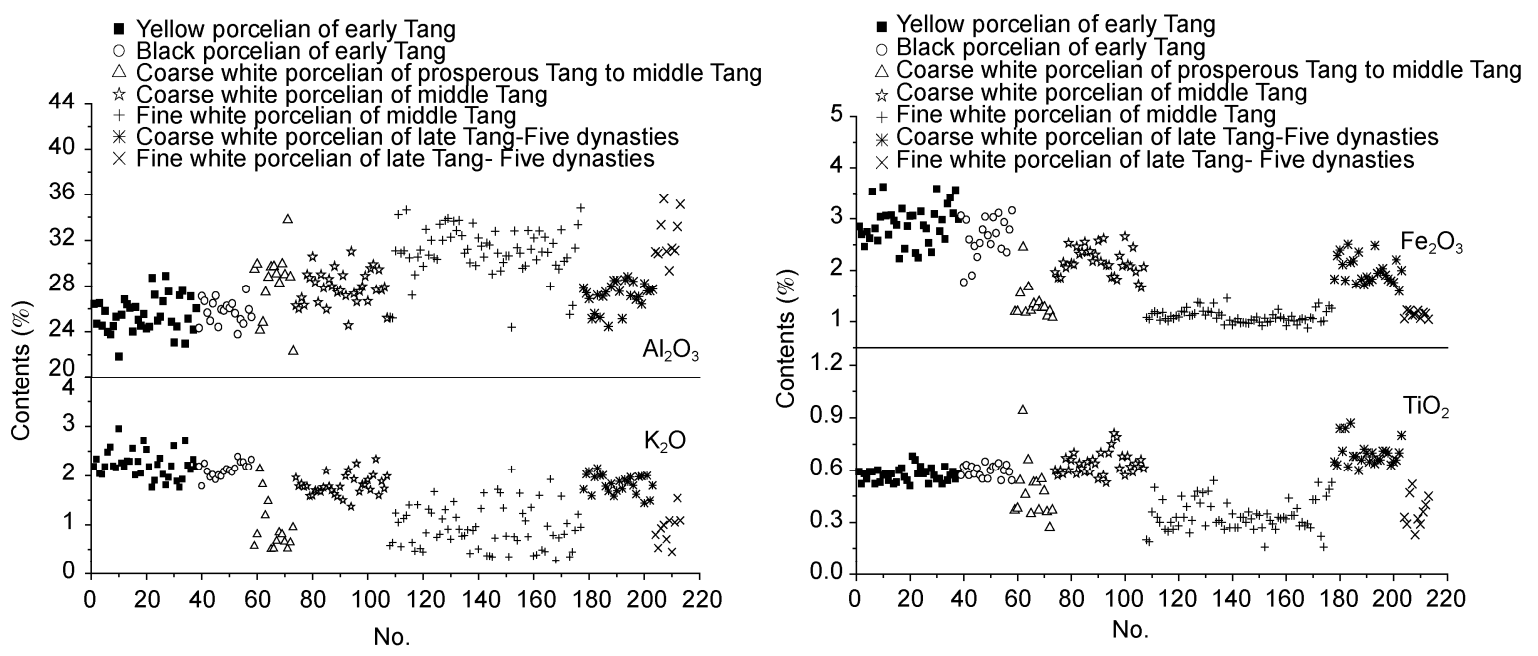

图 10 邢窑唐代瓷胎中 $\mathrm{Al}_{2} \mathrm{O}_{3}, \mathrm{~K}_{2} \mathrm{O}, \mathrm{Fe}_{2} \mathrm{O}_{3}, \mathrm{TiO}_{2}$ 的分析结果
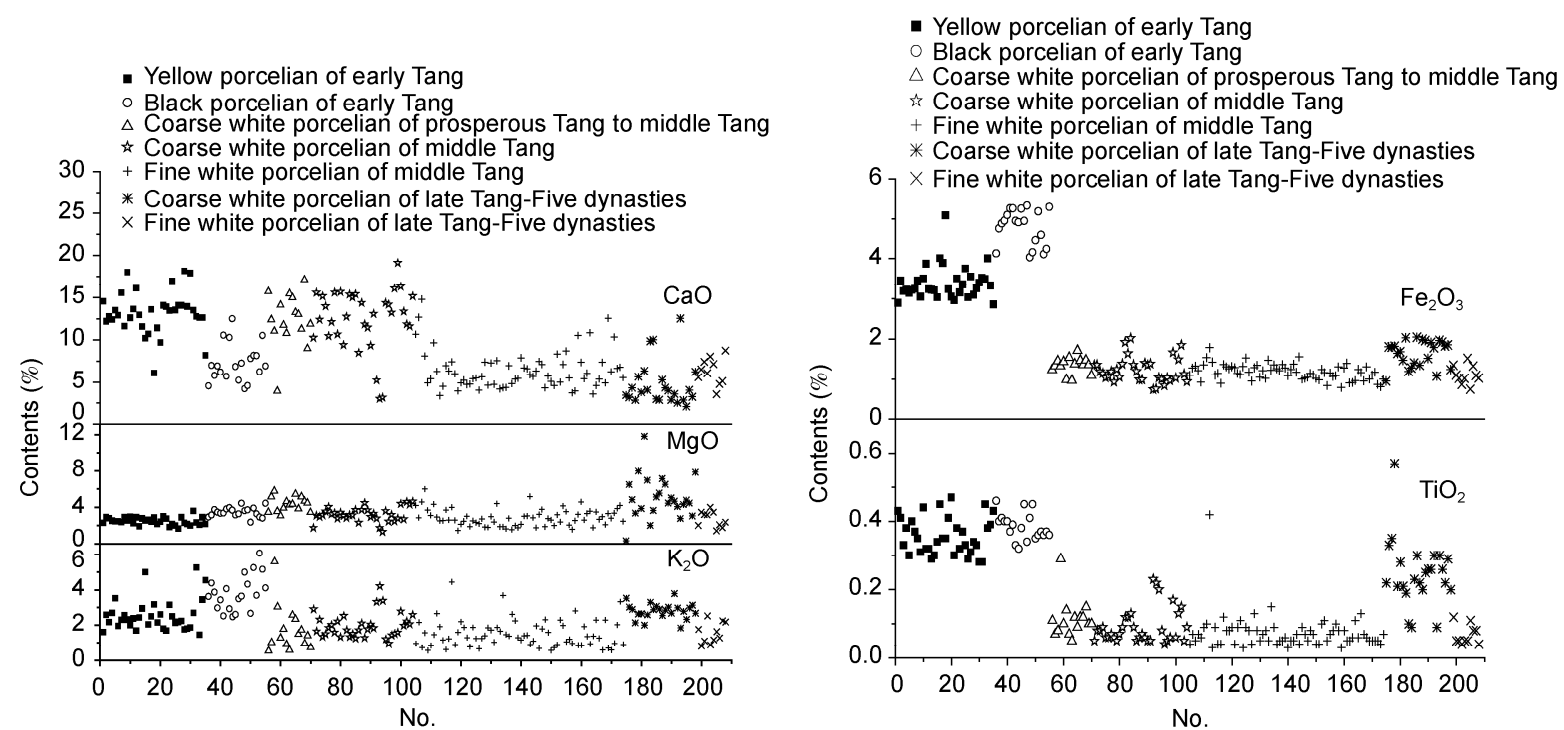

图 11 邢窑唐代瓷釉中 $\mathrm{CaO}, \mathrm{MgO}, \mathrm{K}_{2} \mathrm{O}, \mathrm{Fe}_{2} \mathrm{O}_{3}, \mathrm{TiO}_{2}$ 的分析结果

瓷, 因此它的胎料组成与细白瓷样品还是存在一定 的差别, 但外观上它们胎色较白, 也不施化妆土, 因 此高邑北焦的白瓷胎料有一定的地域性特点. 唐代 细白瓷胎中 $\mathrm{Al}_{2} \mathrm{O}_{3}$ 含量较高, 应该使用了较为优质的 高岭土做胎, 表 9 给出它的胎料与唐代瓷胎上白色化 妆土的主次量化学组成的测试结果, 从数据可以看 出它们组成相对较为接近.

釉料的分析结果表明, 钙质黄釉中 $\mathrm{CaO}$ 含量较 高(13.18\%), $\mathrm{Fe}_{2} \mathrm{O}_{3}$ 含量为 $3.38 \%, \mathrm{TiO}_{2}$ 含量为 $0.36 \%$; 黑釉瓷釉料中 $\mathrm{Fe}_{2} \mathrm{O}_{3}$ 含量更高 $(4.79 \%)$, 这是它呈黑色 的根本原因, 并且 $\mathrm{CaO}$ 含量较低 $(7.27 \%), \mathrm{K}_{2} \mathrm{O}$ 含量相
对较高 $(3.81 \%), \mathrm{TiO}_{2}$ 含量为 $0.39 \%$; 盛唐中粗白瓷与 中唐中粗白瓷釉料相近, 其中 $\mathrm{CaO}$ 含量也较高 (12.45\%), $\mathrm{Fe}_{2} \mathrm{O}_{3}$ 含量为 $1.26 \%, \mathrm{TiO}_{2}$ 含量为 $0.10 \%$; 中 唐细白瓷釉 $\mathrm{CaO}$ 含量较低 $(6.41 \%), \mathrm{Fe}_{2} \mathrm{O}_{3}$ 含量为 $1.18 \%, \mathrm{TiO}_{2}$ 含量为 $0.07 \%$; 晚唐五代中粗白瓷的釉料 组成有所变化, 其 $\mathrm{MgO}$ 含量增加至 $5.05 \%, \mathrm{CaO}$ 含量 低至 $4.72 \%, \mathrm{Fe}_{2} \mathrm{O}_{3}$ 含量增至 $1.65 \%, \mathrm{TiO}_{2}$ 含量增至 $0.25 \%$; 晚唐五代细白瓷釉则与中唐细白瓷釉料组成 相近. 在图 9 中, 黄釉瓷的样品点基本处于中粗白瓷 和黑釉瓷的中间区域，因此黑瓷釉和中粗白瓷釉有 可能是黄釉瓷釉料发展的两个极端方向，在它的基 
表 9 邢窑唐代细白瓷胎及化妆土的主次量化学组成数据 $(\mathrm{wt} \%)$

\begin{tabular}{ccccccccc}
\hline & $\mathrm{Na}_{2} \mathrm{O}$ & $\mathrm{MgO}$ & $\mathrm{Al}_{2} \mathrm{O}_{3}$ & $\mathrm{SiO}_{2}$ & $\mathrm{~K}_{2} \mathrm{O}$ & $\mathrm{CaO}$ & $\mathrm{TiO}_{2}$ & $\mathrm{Fe}_{2} \mathrm{O}_{3}$ \\
\hline $\begin{array}{c}\text { Mean value of fine white porcelain bodies } \\
\text { of the middle Tang dynasty }\end{array}$ & 0.65 & 1.42 & 30.97 & 62.37 & 0.95 & 1.20 & 0.34 & 1.10 \\
$\begin{array}{c}\text { Mean value of fine white porcelain bodies } \\
\text { of the late Tang-Five dynasties }\end{array}$ & 0.63 & 1.10 & 32.20 & 61.28 & 0.92 & 1.37 & 0.37 & 1.15 \\
$\quad$\begin{tabular}{l} 
Mean value of engobe \\
\hline
\end{tabular} & 0.81 & 0.78 & 33.64 & 57.79 & 1.79 & 1.82 & 0.92 & 1.43 \\
\hline
\end{tabular}

础上增加高铁原料就能配出黑釉、减少着色元素(或 增加白色原料) 就能配出透明白釉. 中粗白瓷釉中着 色元素铁、钛含量的明显降低再加上白色化妆土的底 色, 是其呈现白瓷特征的根本原因. 细白瓷由于胎料 较为优质, 胎体本身的白度就很高, 施加透明釉后不 需要化妆土的祄托就能显现白瓷的特征, 而细白瓷 釉的明显特征就是 $\mathrm{CaO}$ 含量的显著降低, 较中唐中 粗白瓷釉中 $\mathrm{CaO}$ 含量降低了一半左右, 同时 $\mathrm{P}_{2} \mathrm{O}_{5}$ 含 量也从 $0.24 \%$ 降低至 $0.13 \%$, 说明其釉灰使用量应该 减少了一半左右. 高邑北焦出土的盛唐-中唐中粗白 瓷虽然胎料较为优质、颜色较白、大多也不施加化妆 土, 但是釉料中 $\mathrm{CaO}$ 含量比中唐细白瓷高出许多, 而 与中唐中粗白瓷相近. 晚唐五代中粗白瓷的釉料工 艺有所变化, 其总体趋势已经与中唐及以前的中粗 白瓷釉有区别, 而更接近于细白瓷釉, 向 $\mathrm{CaO}$ 减少的 方向上变化, 但同时 $\mathrm{MgO}, \mathrm{K}_{2} \mathrm{O}$ 有所增加, 着色元素 铁钛含量明显增加, 说明这个时期普通白瓷的制釉 工艺发生了较为明显的改变.

\subsection{3 烧制工艺与陶瓷性能}

烧成温度测试结果如图 12 所示. 唐早期的䥻质 黄釉瓷烧成温度较低, 波动范围也较大 (1160 $1300^{\circ} \mathrm{C}$, 均值 $\left.1200^{\circ} \mathrm{C}\right)$; 而铅质黄釉胎的烧成温度更 低 $\left(3\right.$ 个样品 $\left.1010^{\circ} \mathrm{C}, 1120^{\circ} \mathrm{C}, 1140^{\circ} \mathrm{C}\right)$, 按照三彩的烧 制工艺, 它还应该是二次烧成, 因为铅釉的温度一般 在 $900^{\circ} \mathrm{C}$ 左右. 唐早期黑釉瓷的烧成温度相对黄釉瓷 为高 (均值 $1290^{\circ} \mathrm{C}$ ). 高邑北焦盛唐-中唐中粗白瓷烧 成温度均值为 $1300^{\circ} \mathrm{C}$, 中唐中粗白瓷的烧成温度均 值为 $1260^{\circ} \mathrm{C}$. 中唐细白瓷的烧成温度均值 $1360^{\circ} \mathrm{C}$, 最高值已超过 $1450^{\circ} \mathrm{C}$, 说明中唐时期的高温窑炉技 术已经达到非常高的水平. 晚唐五代的中粗白瓷的 烧成温度与细白瓷温度相当, 均值都为 $1300^{\circ} \mathrm{C}$.

中唐细白瓷烧成温度很高, 与中粗白瓷差别很 大, 说明应该是专门烧制的, 而不是与其他样品混烧. 从胎体吸水率结果来看, 中唐细白瓷胎的平均吸水

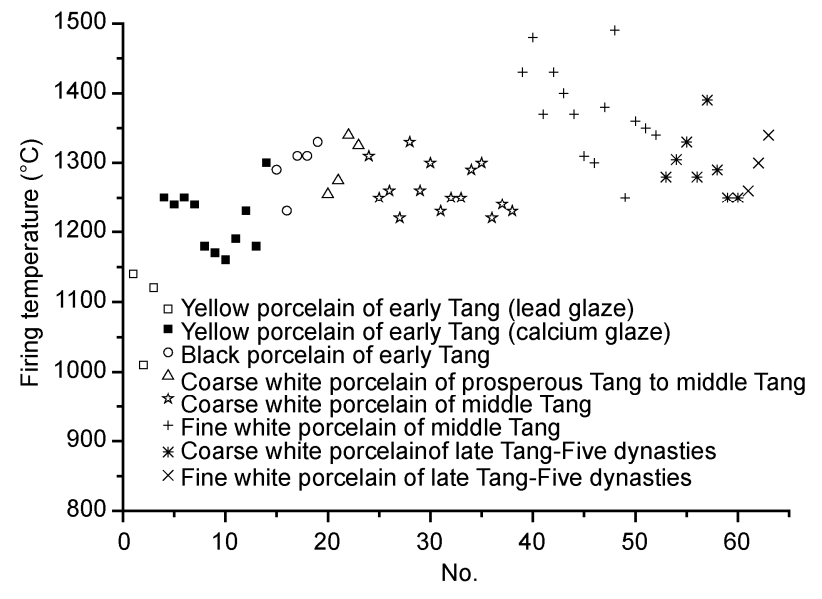

图 12 邢窑唐代瓷器样品的烧成温度

率为 $3.58 \%$, 处于正烧水平(吸水率低于 $1 \%$ ) 的样品烧 成温度基本在 $1450^{\circ} \mathrm{C}$ 左右, 而其平均温度为 $1360^{\circ} \mathrm{C}$, 说明有较多样品属于生烧. 中唐时期细白瓷由于其 胎中 $\mathrm{Al}_{2} \mathrm{O}_{3}$ 含量较高, 而熔剂含量很低, 因此达到正 烧就需要较高的温度, 而其釉中 $\mathrm{CaO}$ 含量的明显降 低应该是古代陶工为了避免由于烧成温度的提高而 造成流釉现象所采取的相应措施. 晚唐五代中粗白 瓷与细白瓷的烧成温度基本一致, 都为 $1300^{\circ} \mathrm{C}$, 说 明它们有可能是同窑烧制, 而瓷器残片的粘连情况 则进一步证实了这一点(如图 13 所示), 所以晚唐五 代细白瓷虽然原料配方与中唐细白瓷相同, 但烧制 工艺已经改变, 不再单独高温烧制而是与中粗白瓷 混烧.

此外, 由于唐代邢窑也是烧制唐三彩的窑厂之 一, 因此在黄嬏瓷中发现低温铅釉就不足为奇了. 这 个现象说明, 唐早期的黄釉瓷可能为有意烧制, 并且 至少存在 2 种工艺, 用高温钲釉制作黄嬏瓷有可能是 为了模仿低温黄釉的特征, 而考古工作者仅凭肉眼 是无法对黄色钻釉和铅釉进行区分的. 唐代低温釉 (三彩)主要是陪葬品, 釉中含有大量的氧化铅, 不适 合作为日用器血, 而高温钻质黄釉较低温铅质黄釉 有较大进步, 其作为盘碗等日用产品无毒副作用, 并 


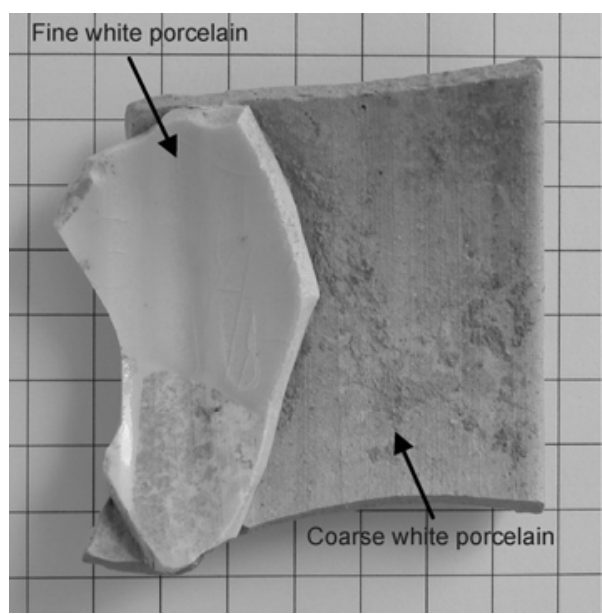

图 13 邢窑临城祁村晚唐 LCH-QC-02-025 样品的外貌照片

且烧成温度可以提高, 以增强胎体的强度和致密度. 另外, 唐代时期青瓷的制作主要是南方的越窑, 所以 邢窑有可能不致力于制作青瓷, 由于北方烧制瓷器 多用氧化焰，从而形成了自己黄釉瓷的特色.

图 14 的色度测试结果表明, 盛唐-中唐中粗白 瓷、中唐中粗白瓷、中唐细白瓷、晚唐五代中粗白瓷

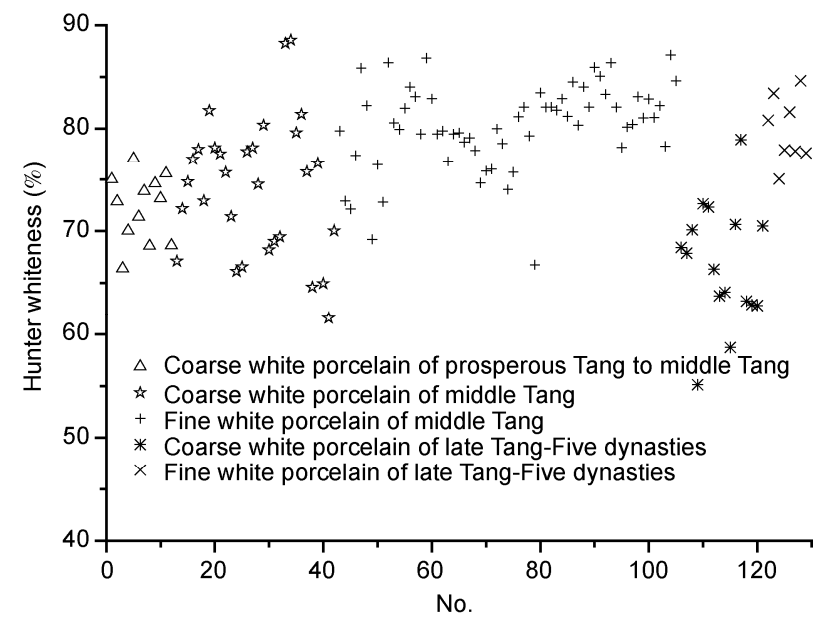

图 14 邢窑唐代白瓷釉面的 Hunter 白度
以及晚唐五代细白瓷釉面 Hunter 白度均值依次变化 为: $72.61 \% \rightarrow 74.08 \% \rightarrow 80.24 \% \rightarrow 66.77 \% \rightarrow 79.85 \%$. 细白瓷的白度较高, 这与其胎釉原料的精选有关. 晚 唐五代中粗白瓷的白度明显降低, 但它们还施加化 妆土, 而前面组成的分析结果指出, 晚唐五代时期中 粗白瓷釉料中着色元素含量明显增加, 说明釉料的 精选及处理程度不高, 这是其白度降低的主要原因. 考古界普遍认为, 邢窑始烧于北朝, 兴盛于隋唐, 至 五代走向衰亡, 而原料和外观白度的测试结果也与 此基本相对应，因此晚唐五代应该是邢窑走向衰落 的转折点.

\section{3 五代及金代样品的测试分析}

\section{1 实验及结果}

\subsection{1 样品的选取}

所有样品及其分期分类信息均由河北省文物研 究所提供, 共选择测试样品 131 件. 样品具体信息见 表 10 , 需要说明的是临城南程村金代中粗白瓷中有 2 件白釉点黑花的样品.

\subsection{2 测试结果}

样品胎釉的化学组成测试结果见表 11. 限于文 章篇幅, 表 11 中只列出了它们的平均值和标准差. 测试结果表明, 白酱双色瓷和白釉点黑花样品胎、白 釉的组成与其他中粗白瓷没有差别, 因此不再单列, 而酱釉和黑花部位的组成单列于表 12 .

\section{2 分析与讨论}

\subsection{1 样品的原料特点及演变}

图 15 和 16 是样品胎和釉中 8 种主次量化学组成 $\left(\mathrm{Na}_{2} \mathrm{O}, \mathrm{MgO}, \mathrm{Al}_{2} \mathrm{O}_{3}, \mathrm{SiO}_{2}, \mathrm{~K}_{2} \mathrm{O}, \mathrm{CaO}, \mathrm{TiO}_{2}, \mathrm{Fe}_{2} \mathrm{O}_{3}\right)$ 的 二维对应分析分析结果. 图 17 和 18 是多元统计分析

\section{表 10 邢窑五代和金代样品信息列表}

\begin{tabular}{|c|c|c|c|c|}
\hline Serial number & Period & Sort & Site & Sum \\
\hline LCH-QC-03-XXX & Five dynasties-Northern Song dynasty & Coarse white porcelain & Qicun & 20 \\
\hline LCH-GT-01-XXX & Five dynasties-Northern Song dynasty & Coarse white porcelain & Gangtou & 20 \\
\hline LCH-CHD-01-XXX & Five dynasties-Northern Song dynasty & Coarse white porcelain & Chengdi & 16 \\
\hline LCH-SHX-01-XXX & Jin dynasty & Coarse white porcelain & Shanxia & 20 \\
\hline LCH-SHX-02-XXX & Jin dynasty & White/brown dual glazed porcelain & Shanxia & 5 \\
\hline LCH-NCHC-01-XXX & Jin dynasty & Coarse white porcelain & Nanchengcun & 35 \\
\hline LCH-CYG-00-XXX & Jin dynasty & Coarse white porcelain & Ciyaogou & 15 \\
\hline
\end{tabular}


表 11 邢窑五代和金代中粗白瓷样品胎、釉的化学组成数据 $(\mathbf{w t} \%)$

\begin{tabular}{|c|c|c|c|c|c|c|c|c|c|c|c|c|c|}
\hline Period & Site & Body/glaze & & $\mathrm{Na}_{2} \mathrm{O}$ & $\mathrm{MgO}$ & $\mathrm{Al}_{2} \mathrm{O}_{3}$ & $\mathrm{SiO}_{2}$ & $\mathrm{~K}_{2} \mathrm{O}$ & $\mathrm{CaO}$ & $\mathrm{TiO}_{2}$ & $\mathrm{Fe}_{2} \mathrm{O}_{3}$ & $\mathrm{MnO}$ & $\mathrm{P}_{2} \mathrm{O}_{5}$ \\
\hline \multirow{6}{*}{$\begin{array}{l}\text { Five dynasties- } \\
\text { Northern Song } \\
\text { dynasty }\end{array}$} & \multirow{2}{*}{ Qicun } & \multirow{2}{*}{ Body } & Mean & 0.36 & 0.79 & 27.16 & 65.45 & 1.80 & 0.72 & 0.70 & 2.03 & 0.02 & 0.03 \\
\hline & & & S.D. & 0.18 & 0.09 & 1.14 & 1.41 & 0.24 & 0.14 & 0.06 & 0.30 & 0.00 & 0.02 \\
\hline & \multirow{2}{*}{ Gangtou } & \multirow{2}{*}{ Body } & Mean & 0.35 & 0.80 & 26.16 & 66.19 & 1.79 & 1.00 & 0.69 & 2.03 & 0.02 & 0.02 \\
\hline & & & S.D. & 0.21 & 0.12 & 1.59 & 1.62 & 0.26 & 0.43 & 0.11 & 0.33 & 0.00 & 0.01 \\
\hline & \multirow{2}{*}{ Chengdi } & \multirow{2}{*}{ Body } & Mean & 0.26 & 0.84 & 26.27 & 65.73 & 1.87 & 1.02 & 0.70 & 2.32 & 0.02 & 0.03 \\
\hline & & & S.D. & 0.13 & 0.07 & 0.97 & 1.10 & 0.27 & 0.23 & 0.12 & 0.18 & 0.00 & 0.00 \\
\hline \multirow{6}{*}{ Jin dynasty } & \multirow{2}{*}{ Shanxia } & \multirow{2}{*}{ Body } & Mean & 0.28 & 0.88 & 32.50 & 58.93 & 3.02 & 0.36 & 1.24 & 1.80 & 0.01 & 0.02 \\
\hline & & & S.D. & 0.13 & 0.12 & 1.03 & 1.09 & 0.38 & 0.06 & 0.14 & 0.16 & 0.00 & 0.00 \\
\hline & \multirow{2}{*}{ Nanchengcun } & \multirow{2}{*}{ Body } & Mean & 0.29 & 0.77 & 30.53 & 61.62 & 2.27 & 0.40 & 0.92 & 2.19 & 0.01 & 0.02 \\
\hline & & & S.D. & 0.15 & 0.09 & 1.00 & 0.91 & 0.29 & 0.05 & 0.09 & 0.10 & 0.00 & 0.00 \\
\hline & \multirow{2}{*}{ Ciyaogou } & \multirow{2}{*}{ Body } & Mean & 0.34 & 0.70 & 30.41 & 61.77 & 1.89 & 0.59 & 0.87 & 2.42 & 0.01 & 0.02 \\
\hline & & & S.D. & 0.13 & 0.09 & 2.29 & 2.15 & 0.15 & 0.11 & 0.11 & 0.27 & 0.00 & 0.00 \\
\hline \multirow{6}{*}{$\begin{array}{l}\text { Five dynasties- } \\
\text { Northern Song } \\
\text { dynasty }\end{array}$} & \multirow{2}{*}{ Qicun } & \multirow{2}{*}{ Glaze } & Mean & 0.59 & 5.24 & 15.90 & 68.08 & 2.73 & 4.46 & 0.24 & 1.76 & 0.03 & 0.12 \\
\hline & & & S.D. & 0.48 & 1.95 & 1.29 & 2.20 & 0.43 & 1.52 & 0.06 & 0.35 & 0.02 & 0.05 \\
\hline & \multirow{2}{*}{ Gangtou } & \multirow{2}{*}{ Glaze } & Mean & 0.56 & 5.37 & 15.35 & 68.54 & 2.66 & 4.61 & 0.24 & 1.69 & 0.02 & 0.11 \\
\hline & & & S.D. & 0.58 & 2.05 & 1.40 & 1.70 & 0.47 & 2.01 & 0.04 & 0.40 & 0.01 & 0.05 \\
\hline & \multirow{2}{*}{ Chengdi } & \multirow{2}{*}{ Glaze } & Mean & 0.41 & 4.22 & 16.55 & 68.65 & 2.71 & 4.36 & 0.32 & 1.78 & 0.02 & 0.11 \\
\hline & & & S.D. & 0.38 & 1.42 & 1.23 & 0.94 & 0.28 & 0.59 & 0.07 & 0.37 & 0.00 & 0.02 \\
\hline \multirow{6}{*}{ Jin dynasty } & \multirow{2}{*}{ Shanxia } & \multirow{2}{*}{ Glaze } & Mean & 0.42 & 4.20 & 16.31 & 68.94 & 3.46 & 4.18 & 0.14 & 1.35 & 0.02 & 0.17 \\
\hline & & & S.D. & 0.31 & 0.91 & 1.08 & 1.23 & 0.44 & 1.53 & 0.06 & 0.12 & 0.00 & 0.06 \\
\hline & \multirow{2}{*}{ Nanchengcun } & \multirow{2}{*}{ Glaze } & Mean & 0.61 & 3.77 & 16.59 & 70.00 & 2.99 & 3.59 & 0.11 & 1.34 & 0.02 & 0.15 \\
\hline & & & S.D. & 0.47 & 0.86 & 1.33 & 1.08 & 0.31 & 1.22 & 0.05 & 0.17 & 0.00 & 0.09 \\
\hline & \multirow{2}{*}{ Ciyaogou } & \multirow{2}{*}{ Glaze } & Mean & 0.46 & 1.95 & 16.03 & 70.89 & 3.62 & 3.86 & 0.33 & 1.86 & 0.02 & 0.31 \\
\hline & & & S.D. & 0.31 & 0.79 & 1.86 & 2.52 & 0.45 & 2.18 & 0.13 & 0.13 & 0.01 & 0.12 \\
\hline
\end{tabular}

表 12 邢窑金代白酱双色和白釉黑花样品中酱釉和黑花部位的化学组成数据 $(\mathrm{wt} \%)$

\begin{tabular}{|c|c|c|c|c|c|c|c|c|c|c|c|}
\hline Serial number & Sort & $\mathrm{Na}_{2} \mathrm{O}$ & $\mathrm{MgO}$ & $\mathrm{Al}_{2} \mathrm{O}_{3}$ & $\mathrm{SiO}_{2}$ & $\mathrm{~K}_{2} \mathrm{O}$ & $\mathrm{CaO}$ & $\mathrm{TiO}_{2}$ & $\mathrm{Fe}_{2} \mathrm{O}_{3}$ & $\mathrm{MnO}$ & $\mathrm{P}_{2} \mathrm{O}_{5}$ \\
\hline LCH-SHX-02-001 & \multirow{5}{*}{$\begin{array}{l}\text { White/brown dual glazed } \\
\text { porcelain }\end{array}$} & 0.49 & 2.36 & 16.04 & 68.21 & 4.35 & 3.45 & 0.50 & 3.60 & 0.03 & 0.07 \\
\hline LCH-SHX-02-002 & & 1.06 & 2.42 & 15.20 & 67.85 & 4.08 & 4.16 & 0.48 & 3.75 & 0.04 & 0.09 \\
\hline LCH-SHX-02-003 & & 0.16 & 3.47 & 13.73 & 67.95 & 3.28 & 6.59 & 0.35 & 3.47 & 0.04 & 0.09 \\
\hline LCH-SHX-02-004 & & 1.40 & 4.06 & 15.06 & 65.28 & 4.03 & 5.05 & 0.41 & 3.70 & 0.04 & 0.12 \\
\hline LCH-SHX-02-005 & & 0.25 & 2.55 & 17.54 & 65.28 & 3.79 & 4.74 & 0.76 & 4.09 & 0.03 & 0.05 \\
\hline LCH-NCHC-01-053 & \multirow{2}{*}{$\begin{array}{l}\text { White porcelains with } \\
\text { underglaze decoration of black } \\
\text { flowers }\end{array}$} & 0.83 & 3.17 & 13.30 & 69.05 & 2.87 & 5.92 & 0.33 & 3.53 & 0.04 & 0.06 \\
\hline LCH-NCHC-01-055 & & 0.95 & 3.57 & 13.27 & 67.47 & 3.09 & 6.93 & 0.31 & 3.42 & 0.05 & 0.12 \\
\hline
\end{tabular}

中权重较大的因子组成的单元素散布分析. 从表 11 的数据可以看出, 五代北宋 3 个窑址出土中粗白瓷的 胎釉组成基本相似, 因此统计分析图中五代北宋样 品不再按窑址单列; 金代 3 个窑址胎釉之间存在差异, 将它们按窑址单列. 另外, 邢窑唐代样品的分析指出, 晚唐五代时期中粗白瓷的釉料特点发生了显著变化, 而胎料与中唐中粗白瓷相比基本无变化, 因此在图 15 至 18 样品的统计分析中, 对胎料的分析加入晚唐 五代中粗白瓷的样品数据, 对釉料的分析加入中唐 和晚唐五代中粗白瓷的样品数据(见表 8), 以此来更 好地反映邢窑在五代之后的发展规律.

从胎的统计分析结果可以看出, 五代北宋与晚
唐五代时期中粗白瓷的胎料基本相似，五代北宋的 样品点更为分散, 而金代中粗白瓷的胎料组成明显 有所变化, 其 $\mathrm{Al}_{2} \mathrm{O}_{3}$ 含量 $(31.16 \%)$ 明显高于晚唐五代 和五代北宋中粗白瓷样品 (26.86\%); 临城山下金代中 粗白瓷的胎明显不同于临城南程村和临城磁窑沟金 代的中粗白瓷, 其 $\mathrm{TiO}_{2}$ 含量(1.24\%)较高, 而 $\mathrm{Fe}_{2} \mathrm{O}_{3}$ 含量 $(1.80 \%)$ 较低, $\mathrm{Al}_{2} \mathrm{O}_{3}$ 含量 $(32.50 \%)$ 和 $\mathrm{K}_{2} \mathrm{O}$ 含量 (3.02\%)也较高, 说明临城山下中粗白瓷选用胎料存 在一定的地域性特点.

从釉的分析结果可以看出, 邢窑五代北宋中粗 白瓷的制釉工艺延续了自晚唐五代开始转变的特点, 与中唐时期中粗白瓷相比, 它们釉中 $\mathrm{CaO}$ 含量也明 


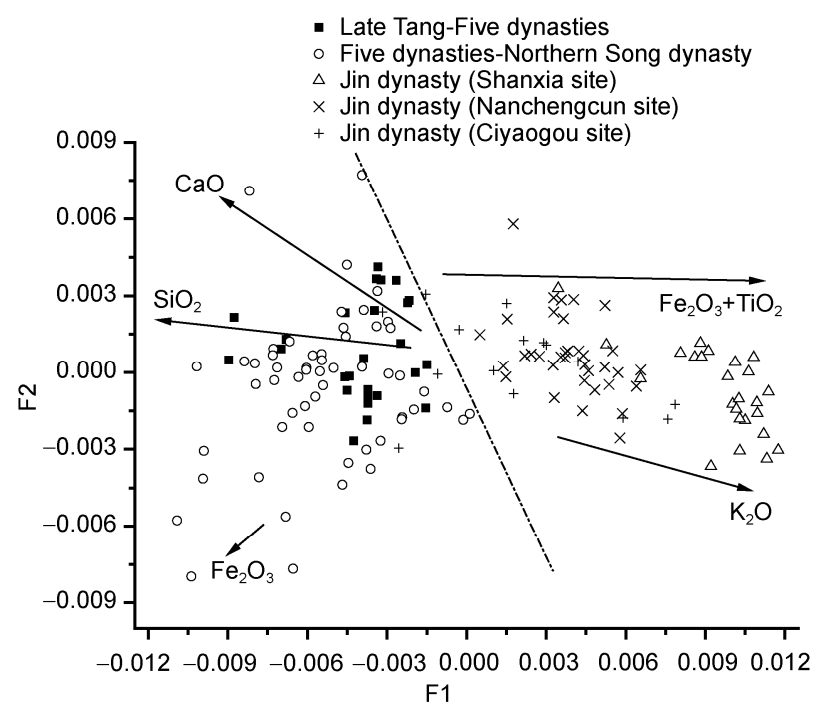

图 15 邢窑晚唐五代至金代中粗白瓷胎的二维对应分析结果

显降低, $\mathrm{MgO}$ 含量也较高, 由钻釉向镁钻釉转变, 而 着色元素铁钛含量也有所增加, 并且釉的组成较为 分散; 金代中粗白瓷釉中 $\mathrm{CaO}$ 含量与五代北宋相似, $\mathrm{MgO}$ 含量又有所降低, 临城山下和临城南程村出土 中粗白瓷釉中铁钛含量比五代时期有所下降; 但临 城磁窑沟的铁钛含量又有所增加, 其配方工艺与山 下和南程村有所不同, 并且从外观釉层上可以明显 看出磁窑沟的中粗白瓷比山下和南程村的质量差, 说明磁窑沟中粗白瓷的釉料精选和处理程度较低.

金代时期的中粗白瓷中有一些白酱双色、白釉黑
花的品种, 白酱双色的制作是对器物的内外表面分 别施加了白釉和酱釉所形成的，而白釉黑花是在胎 体上先用黑釉点出花瓣造型, 再施加透明白釉烧成 的. 从表 12 的数据可以看出, 黑釉中 $\mathrm{Fe}_{2} \mathrm{O}_{3}$ 和 $\mathrm{TiO}_{2}$ 含量 $(3.71 \%, 0.44 \%)$ 比白釉 $(1.35 \%, 0.12 \%)$ 明显较高, $\mathrm{SiO}_{2}$ 含量 $(67.47 \%$ )比白釉(69.59\%)降低, 其他组成基 本相近.

\subsection{2 烧制工艺与陶瓷性能}

烧成温度的测试结果见图 19. 五代北宋中粗白瓷 的烧成温度均值为 $1305^{\circ} \mathrm{C}$, 与晚唐五代相似; 金代 中粗白瓷的烧成温度有所提高, 均值为 $1335^{\circ} \mathrm{C}$,

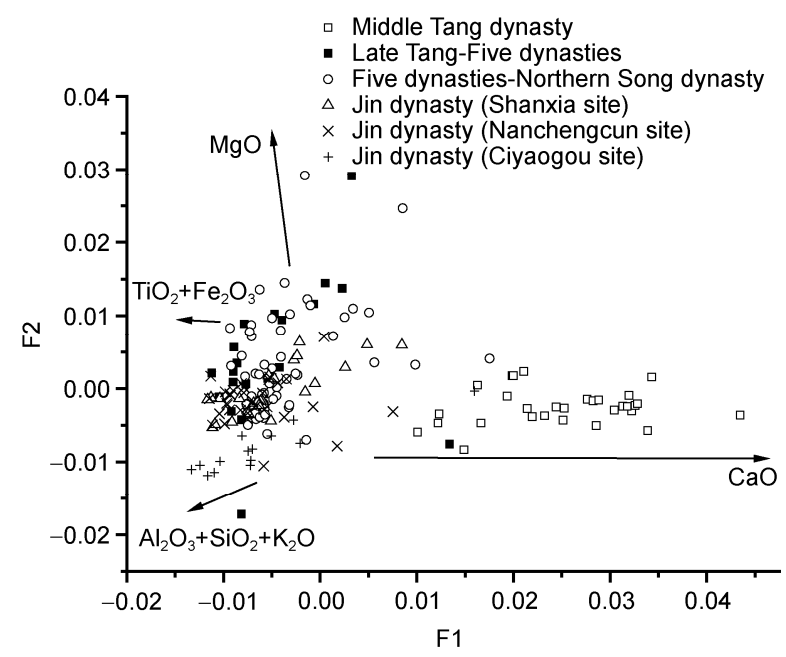

图 16 邢窑中唐至金代中粗白瓷釉的二维对应分析结果
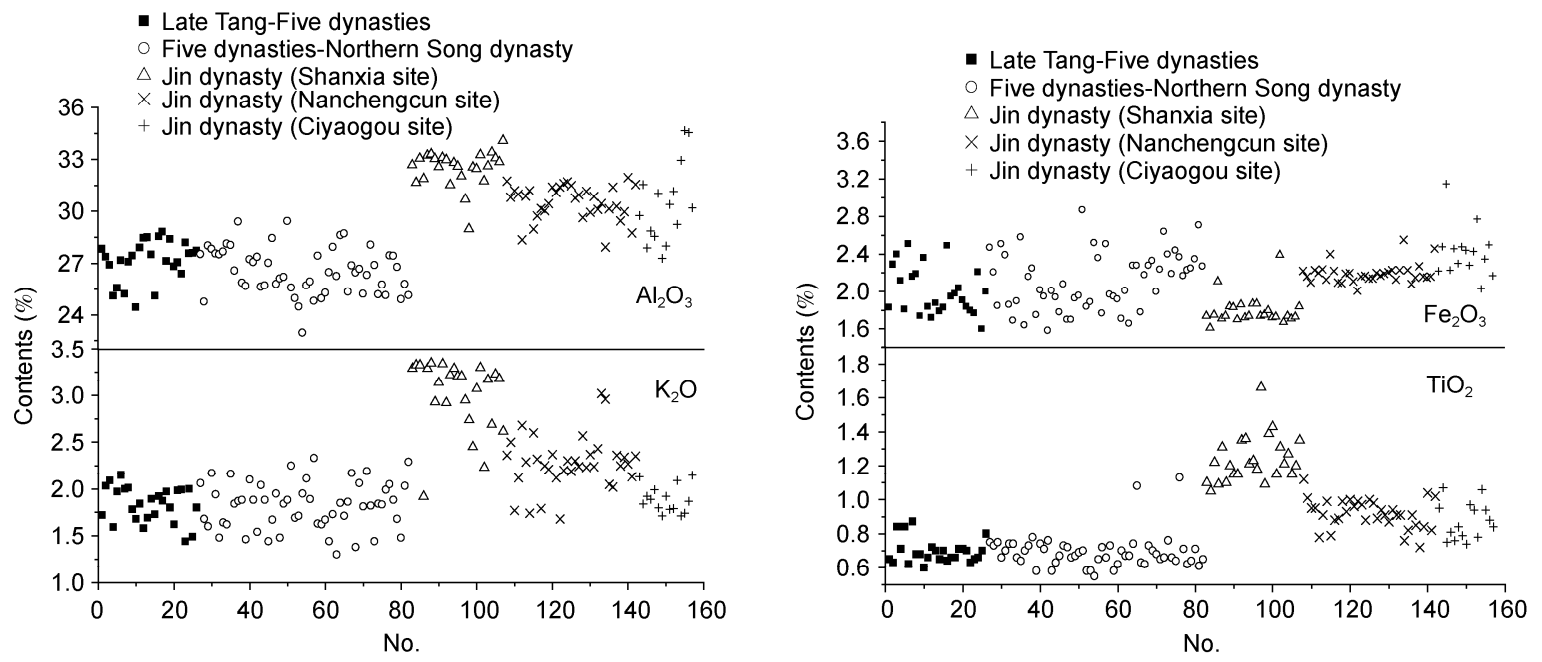

图 17 邢窑晚唐五代至金代中粗白瓷胎中 $\mathrm{Al}_{2} \mathrm{O}_{3}, \mathrm{~K}_{2} \mathrm{O}, \mathrm{Fe}_{2} \mathrm{O}_{3}, \mathrm{TiO}_{2}$ 的分析结果 

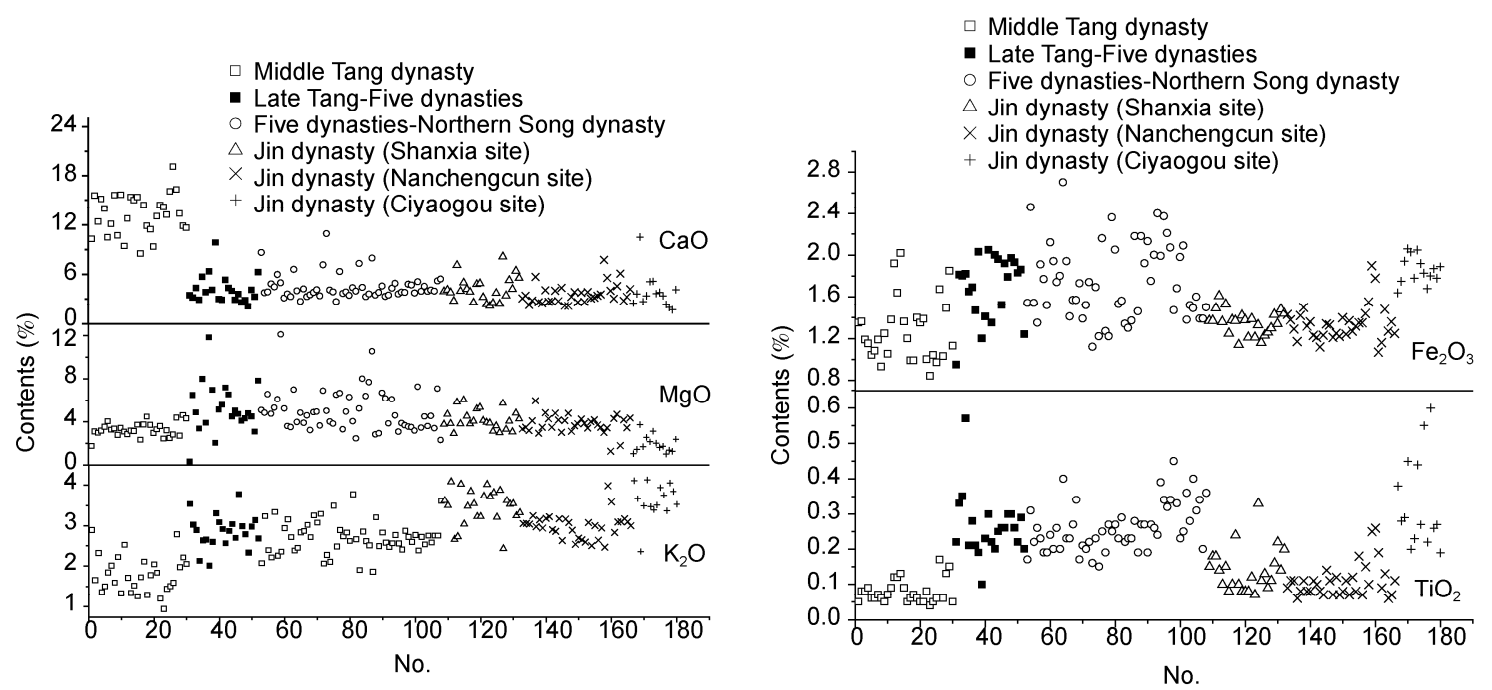

图 18 邢窑中唐至金代中粗白瓷釉中 $\mathrm{CaO}, \mathrm{MgO}, \mathrm{K}_{2} \mathrm{O}, \mathrm{Fe}_{2} \mathrm{O}_{3}, \mathrm{TiO}_{2}$ 的分析结果

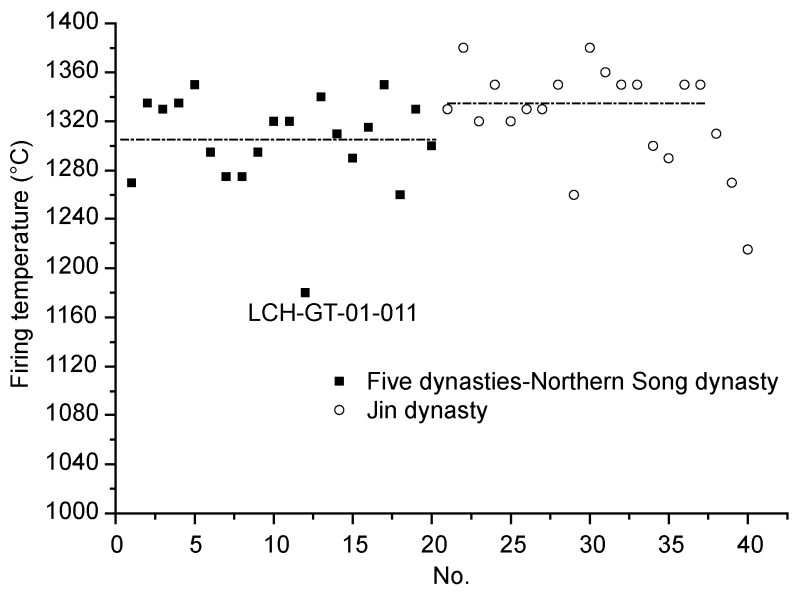

图 19 邢窑五代及金代样品瓷胎的烧成温度

磁窑沟金代中粗白瓷的烧成温度较低(图 19 中最后 3 个样品).

图 19 的测试结果表明, 临城岗头五代北宋中粗 白瓷样品中 LCH-GT-01-011, 它的烧成温度是 $1180^{\circ} \mathrm{C}$, 明显低于其他样品. 这件样品外观看上去属 于生烧或素烧样品, 直观上看不出有釉层的痕迹, 而 化妆土痕迹非常明显. 对其表面进行化学组成测试, 结果表明它的表面已经上了一层釉 $(\mathrm{MgO} 5.23 \%, \mathrm{CaO}$ 7.29\%). 用小刀刮这层釉, 质地较硬, 说明釉层也已 经烧过，只不过当时没有玻璃化. 我们将其切割开来, 并将其中一部分置于高温电炉中, 烧至 $1300^{\circ} \mathrm{C}$, 冷 却后观察, 它已经形成了非常好的釉层, 并且颜色与 其他中粗白瓷没有差别, 如图 20 所示, 这也同时说
明当时烧制白瓷应该是在一般的氧化气氛下烧成的. 另外, 我们测试的唐早期钙质黄釉瓷中有些样品的 烧成温度只有 $1160^{\circ} \mathrm{C}$, 但也成了釉, 这就说明自晚 唐五代以来，由于中粗白瓷釉中 $\mathrm{CaO}$ 含量的明显降 低, 熔剂含量的减少, 对釉的玻璃化温度提出了更高 的要求.

另外，金代临城山下和南程村的中粗白瓷胎体 的吸水率普遍较低( $0.9 \%)$, 基本都达到了正烧水平, 这甚至超过了中唐时期细白瓷的平均水平; 金代中 粗白瓷胎体的抗折强度最高达 $98.3 \mathrm{MPa}$, 这也是我 们测试邢窑样品中最高的强度. 金代中粗白瓷胎中 $\mathrm{Al}_{2} \mathrm{O}_{3}$ 含量也较高 $(31.16 \%)$, 与中唐时期的细白瓷相 近, 但是中唐的细白瓷需要 $1450^{\circ} \mathrm{C}$ 的高温才能正烧,

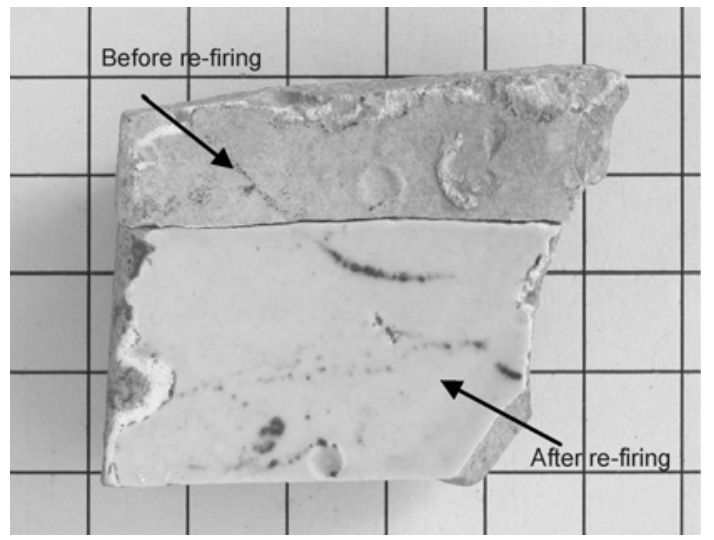

图 20 临城岗头五代北宋 LCH-GT-01-011 样品重烧前后 
而它的正烧温度在 $1350^{\circ} \mathrm{C}$ 左右, 这是由于金代胎中 $\mathrm{K}_{2} \mathrm{O}$ 含量 $(3 \%)$ 要高于中唐细白瓷 $(0.9 \%)$, 这有助于降 低胎体的烧结温度.

图 21 釉面白度的测试结果表明, 邢窑五代北宋 和金代样品的白度值接近, 均值都为 66.75 , 而金代 样品白度值分布较为集中一些, 而五代北宋样品波 动性较大. 五代北宋样品仍然使用化妆土, 釉料工艺 延续了晚唐五代的特点, 由于它们瓷釉中着色元素 铁钛含量较高, 因此釉面白度值较低; 金代样品大多 不施化妆土, 但是金代样品瓷釉中铁钛含量较五代 北宋时期有所降低, 因此即使没有白色化妆土的祄 托，它们的白度值却与五代时期基本相当. 另外，金 代中粗白瓷与五代北宋相比, 胎中着色元素 $\mathrm{Fe}_{2} \mathrm{O}_{3}$ 含 量没有明显降低, $\mathrm{TiO}_{2}$ 含量反而有所增加, 因此大多 数金代中粗白瓷样品都显现灰青色, 是由于没有施 加化妆土而直接反映出了胎体的颜色之故.

结合物理性能的测试结果来看, 金代样品虽然 白度也不高, 但是胎体的烧结程度很好, 说明这个时 期不重视瓷器的外观白度, 而对胎体的内在质量有 了更高的要求. 但不可否认的是, 金代的制釉工艺应 该是对邢窑晚唐五代以来的一种承继和发展, 而金 代中粗白瓷的胎料明显有所变化, 使用了 $\mathrm{Al}_{2} \mathrm{O}_{3}$ 含量 较高的原料, 与中唐时期细白瓷胎高铝含量特征相 近, 但它们原料中着色元素铁钛含量较多, 原料本身 的白度不高, 没有达到中唐细白瓷胎的水平. 但它们 胎质较细, 说明金代对原料的处理程度相对较为精 细, 这也是它们能在 $1350^{\circ} \mathrm{C}$ 就能普遍达到正烧的原 因之一, 因为原料的精细程度与化学组成、烧成温度

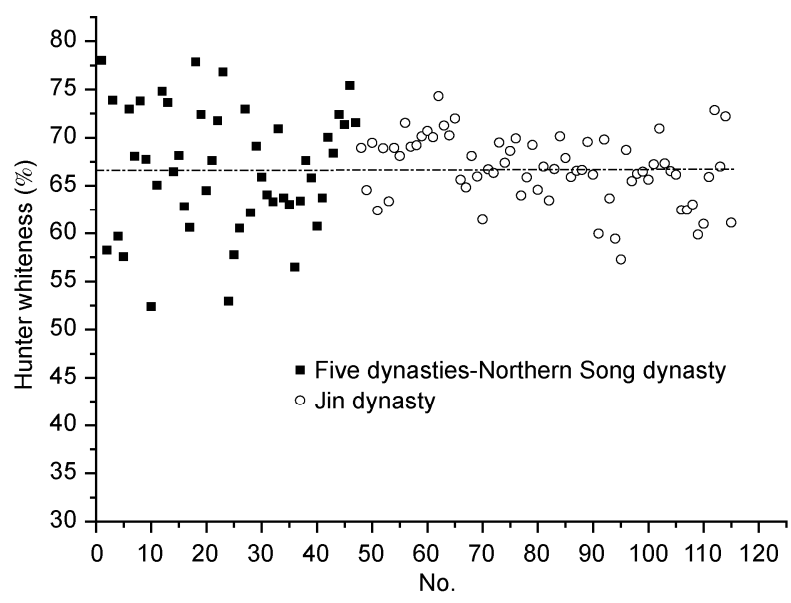

图 21 邢窑五代及金代瓷器釉面的 Hunter 白度值
一样都是影响胎体烧结致密的关键因素.

目前考古界认为, 邢窑兴盛于隋唐, 至五代衰落, 而金代时期邢窑地区的烧造则基本属于定窑系产品. 从我们的分析来看, 邢窑晚唐五代时期的中粗白瓷 的制釉工艺开始发生了变化, 而五代北宋以及金代 的制釉工艺应该是对晚唐时期变革的一种延续和发 展. 但是, 晚唐和五代时期中粗白瓷胎釉的组成都非 常分散, 釉中着色元素含量也较高, 这应该是其作为 转变期的一种反映, 也可能同样反映了当时的社会 动乱情况. 金代的样品点则相对较为集中, 说明金代 的胎釉制作、配方工艺又逐渐稳定下来. 另外, 邢窑 五代北宋的中粗白瓷延续了隋唐时期的工艺特点, 胎体较粗粘, 靠施加化妆土来改善胎体表面的质量 和增白呈色, 而金代样品则大多不施加化妆土, 并且 胎质相对较细、胎体较薄, 从瓷器的制作技法上已经 脱离了邢窑隋唐、五代粗白瓷的风格.

\section{4 总结}

（1）邢窑隋代白瓷的发展脉络非常清晰. 隋代中 粗白瓷与青瓷的最大差别在于对釉料的选择和处理, 使得着色元素减少，透明釉加上白色化妆土的底色， 首先呈现了白釉瓷的基本特征. 第一类细白瓷则基 本是以精细的白色化妆土原料为胎体, 釉料是在中 粗白瓷釉料配方的基础上, 或减少釉灰的使用量, 或 引入高钾原料; 第二类细白瓷是在第一类细白瓷基 础上, 胎釉中都大量引入高钾原料以及釉中掺入一 定量石英的结果. 透影白瓷与第二类细白瓷原料特 征相似, 区别在于古代陶工对器物的胎体进行了刻 意的减薄加工，使其达到了透光的效果，是制瓷工艺 不断改进的结晶. 可以看出, 邢窑隋代白瓷的发展集 中体现了我国古代劳动人民的伟大智慧与创新精神, 透影白瓷的烧制成功就是古代发明创造的典范, 其 开创了我国使用碱钻釉的先河, 是我国陶瓷工艺史 上的伟大创举.

(2) 邢窑唐代早期钙质黄釉瓷的大量出现应该 是受唐三彩黄釉的影响而制造的有自己特色的一类 产品, 并且它们比三彩黄釉更具有实用性. 另外, 唐 代特别是中唐时期是邢窑的鼎盛时期, 其高温窑炉 技术取得重大突破，使得中唐细白瓷产量很大，已成 为 “天下无贵贱通用之物”，但是它们中相当一部分 样品也还是生烧的, 因为达到正烧需要 $1450^{\circ} \mathrm{C}$, 这 
也是我们迄今发现中国古陶瓷中最高的烧成温度. 邢窑唐代中粗白瓷与细白瓷的分类关系可以依靠是 否施加化妆土以及胎料中 $\mathrm{Fe}_{2} \mathrm{O}_{3}$ 的含量作为区分标准. 唐代中粗白瓷由于胎体原料中着色元素铁钛含量较 高, 仍然需要依靠化妆土来呈色; 而细白瓷产品胎釉 原料较为优质, 外观白度也较高, 釉料中 $\mathrm{CaO}$ 含量比 中粗白瓷明显降低. 晚唐五代时期是邢窑普通白瓷 制釉工艺的转折点, 其外观白度也开始降低, 这可能 与晚唐五代时期的社会动乱情况有关; 晚唐五代细 白瓷与中粗白瓷为同窑烧制, 这与中唐时期的情形 截然不同。

（3）邢窑五代北宋时期的发展基本是对晚唐五 代的延续. 金代的邢窑已经不同于隋唐时期的邢窑,
这个时期制作技法发生了变化, 普通白瓷的制作也 比较注重于制品的内在品质, 胎料中 $\mathrm{Al}_{2} \mathrm{O}_{3}$ 含量较高, 外观白度虽然不高, 但胎体烧结程度较好, 考古界都 把它们认为是定窑系产品, 但金代的制釉工艺应该 是对中粗白瓷釉料配方自晚唐五代开始变革的继承 和发展, 釉中 $\mathrm{CaO}$ 含量明显降低. 其实, 隋代细白瓷 和透影白瓷已经是减少釉中 $\mathrm{CaO}$ 含量的先行者, 并 且透影白瓷由于大幅度增加了 $\mathrm{K}_{2} \mathrm{O}$ 的含量直接开创 了碱钻釉的先河, 而唐代细白瓷的大量生产可能进 一步扩大了这种影响, 使得晚唐五代时期普通白瓷 (中粗白瓷)制釉工艺发生了明显的改变, 并一直延续 到了金代.

\section{参考文献}

1 王会民, 张志忠. 邢窑调查试掘的主要收获. 见: 邢窑遗址研究. 北京: 科学出版社, 2007. 37-47

2 王会民, 焚书海, 张志忠. 邢窑遗址调查、试掘报告. 见: 邢窑遗址研究. 北京: 科学出版社, 2007. 60-112

3 张志忠, 王会民. 邢窑隋代透影白瓷. 见: 邢窑遗址研究. 北京: 科学出版社, 2007. 356-361

4 河北省邢窑研究组. 邢窑工艺技术研究. 河北陶瓷, 1987, (2): 6-26

5 陈尧城, 张福康, 张志忠, 等. 邢窑隋唐细白瓷研究. 景德镇陶瓷学报, 1990, 11 (1): 45-53

6 张志刚, 李家治. 邢窑白瓷化学组成及工艺的研究. 景德镇陶瓷学院学报, 1992, 13(1): 15-29

7 冯向前, 冯松林, 徐清, 等. 河北三大白瓷名窑精细白瓷的 SRXRF 无损分析及界定标准的初步研究. 核技术, 2002, 25(10): 827-831

8 凌雪, 冯敏, 胡耀武, 等. 邢窑、定窑和巩窑白瓷显微结构的对比分析. 电子显微学报, 2004, 23(2): 188-193

9 杨文山, 张爱茹. 邢窑白瓷分类与工艺研究. 中国历史文物, 2007, 4: 56-65

10 朱铁权, 王昌燧, 张尚欣, 等. 隋代邢窑粗白瓷胎料配方研究. 岩石矿物学杂志, 2010, 29(3): 313-318

11 罗宏杰. 中国古陶瓷与多元统计分析. 北京: 中国轻工业出版社, 1997. 12-38

12 李家治, 主编. 中国科学技术史·陶瓷卷. 北京: 科学出版社, 1998. 161-163 